\title{
Neural networks for relational learning: an experimental comparison
}

\author{
Werner Uwents • Gabriele Monfardini • \\ Hendrik Blockeel • Marco Gori • Franco Scarselli
}

Received: 10 March 2007 / Revised: 10 June 2010 / Accepted: 17 June 2010 / Published online: 24 July 2010 (C) The Author(s) 2010

\begin{abstract}
In the last decade, connectionist models have been proposed that can process structured information directly. These methods, which are based on the use of graphs for the representation of the data and the relationships within the data, are particularly suitable for handling relational learning tasks. In this paper, two recently proposed architectures of this kind, i.e. Graph Neural Networks (GNNs) and Relational Neural Networks (RelNNs), are compared and discussed, along with their corresponding learning schemes. The goal is to evaluate the performance of these methods on benchmarks that are commonly used by the relational learning community. Moreover, we also aim at reporting differences in the behavior of the two models, in order to gain insights on possible extensions of the approaches. Since RelNNs have been developed with the specific task of learning aggregate functions in mind, some experiments are run considering that particular task. In addition, we carry out more general experiments on the mutagenesis and the biodegradability datasets, on which several other relational learners have been evaluated. The experimental results are promising and suggest that RelNNs and GNNs can be a viable approach for learning on relational data.
\end{abstract}

Editors: Thomas Gärtner and Gemma Garriga.

W. Uwents · H. Blockeel

Department of Computer Science, Katholieke Universiteit Leuven, Leuven, Belgium

W. Uwents

e-mail: werner.uwents@cs.kuleuven.be

H. Blockeel

e-mail: hendrik.blockeel@cs.kuleuven.be

G. Monfardini · M. Gori · F. Scarselli (凶)

Dipartimento di Ingegneria dell'informazione, Università di Siena, Siena, Italy

e-mail: franco@dii.unisi.it

G. Monfardini

e-mail: monfardini@dii.unisi.it

M. Gori

e-mail: marco@dii.unisi.it 
Keywords Relational learning · Graph neural networks · Relational neural networks · Mutagenesis · Biodegradability

\section{Introduction}

Object localization (Bianchini et al. 2005), image classification (Francesconi et al. 1998), natural language processing (Krahmer et al. 2003), bioinformatics (Baldi and Pollastri 2004), QSAR (Micheli et al. 2001), web page scoring, social network analysis (Newman 2001) and relational learning are examples of application domains where the information of interest is encoded into a set of basic entities and relationships between them. In all these domains, the data is naturally represented by sequences, trees, and, more generally, directed or undirected graphs. In fact, nodes can denote concepts while edges can specify their relationships. A machine learning technique for graphical domains is formally described as a function $\varphi$, to be learned by examples, that computes a value $\varphi(\boldsymbol{G}, n)$, where $\boldsymbol{G}$ is a graph and $n$ one of its nodes. Intuitively, $\varphi(\boldsymbol{G}, n)$ is a property of the concept $n^{1}$ that is predicted using all the known concepts and their relationships.

For example, relational databases contain information that is naturally encoded as graphs: each tuple of a relation can be denoted by a node, while the relationships between different tuples are represented by edges (see Fig. 1). The nodes of the graph have labels (i.e., feature vectors of real numbers) which correspond to the fields of the tuples. Recently, the study of

\begin{tabular}{|c|c|c|c|c|c|c|c|c|c|c|}
\hline name & address & sex & rating & $i d$ & customer_ic & type & id & Accounts_id & In/out & Quantity \\
\hline Mr A & Rome & Male & 2 & & & plain & & $\rightarrow$ & out & 10,000 \\
\hline Mr B & Siena & Male & $?$ & & & saving & & & in & 1,000 \\
\hline Mrs B & London & Female & 2 & & & saving & & & out & 30,000 \\
\hline & $:$ : & & $::$ & $::$ & $:$ : & $:$ : & $:$ : & : : & $:$ : & : : \\
\hline
\end{tabular}

Customers

Accounts

Transactions

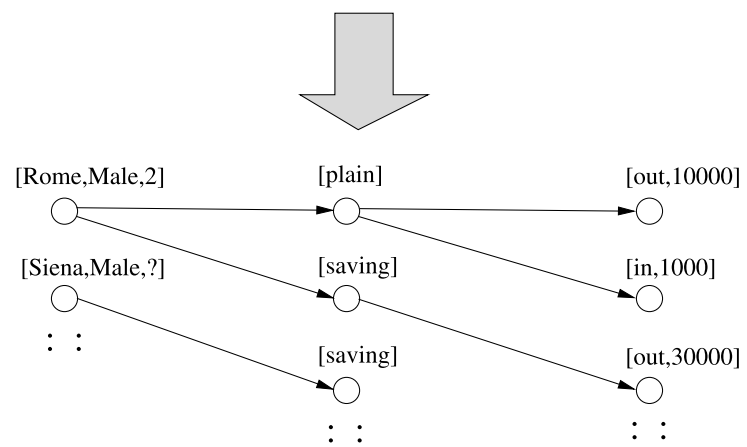

Fig. 1 A relational database and its graphical representation. Tuples are represented by nodes and fields by node labels. The goal of a relational learner is to predict the unknown value of a tuple field (here represented by a question mark)

\footnotetext{
${ }^{1}$ For sake of simplicity, in this paper, the formally correct sentences "the concept represented by $n$ " and "the relationship represented by $(n, u)$ " are sometimes shortened to "the concept $n$ " and "the relationship $(n, u)$," respectively.
} 
machine learning techniques for relational data has received an increasing interest from researchers. A common goal consists of predicting the value of a field, i.e. learning a function $\varphi(\boldsymbol{G}, n)$ from examples, that takes as input a database $\boldsymbol{G}$ and a tuple $n$ and returns the value of a target field of $n$. In the case of Fig. 1, $\varphi$ may be used to predict the customer ratings: $\varphi$ exploits all the database information for the prediction; the training set consists of customers that have or have not payed their debts.

In the last years, new connectionist models were proposed that are capable to take in input graphs and trees directly, embedding the relationships between nodes into the processing scheme (Hammer and Jain 2004). They extend support vector machines (Kondor and Lafferty 2002; Gärtner 2003), neural networks (Sperduti and Starita 1997; Frasconi et al. 1998; Gori et al. 2005) and SOMs (Hagenbuchner et al. 2003) to structured data. The main idea underlying those methods is to automatically obtain an internal flat representation of the symbolic and subsymbolic information collected in the graphs.

In this paper, we focus on supervised learning and we discuss and experimentally evaluate two connectionist models that have been recently proposed, i.e. Relational Neural Networks (RelNNs) (Blockeel and Bruynooghe 2003; Uwents and Blockeel 2005) and Graph Neural Networks (GNNs) (Gori et al. 2005). Those models are peculiar for two different reasons. RelNNs have been defined having relational learning in mind and their characteristics are specifically designed to obtain a good performance on tasks from such a field. On the other hand, the GNN model has been conceived to be general and to be able to process directly, i.e., without a pre-processing, a very large class of graphs, including for instance, cyclic and undirected graphs.

The paper experimentally evaluates the performance of those methods on benchmarks that are commonly adopted by the relational learning community. The results are promising and are comparable to the state of the art on benchmarks on QSAR problems, suggesting that RelNNs and GNNs can be a viable approach for learning on relational data. Moreover, we study and report differences in the behavior of the two models, in order to have insights on the possible extensions of the approaches. Actually, RelNNs and GNNs differ for the connectionist components they exploit, for the kind of graphs they can process and for the learning algorithm. The analysis of the experimental results aims to evaluate how each difference affects the performance and, more generally, the capabilities of the two models.

The paper is organized as follows. In the next section, we review RelNNs, GNNs and some literature on connectionist models for graph processing. Section 3 describes the experimental comparison. Finally, the conclusions are drawn in Sect. 4.

\section{Graph processing by neural networks}

There exists an extensive literature on the application of neural networks to structured data domains. In this section, a quick overview of a number of approaches is given and GNNs and RelNNs are situated with regard to other methods. In order to reach such a goal, we introduce a general framework that is useful to formally describe the considered techniques.

In the following, a graph $\boldsymbol{G}$ is a pair $(\boldsymbol{N}, \boldsymbol{E})$, where $\boldsymbol{N}$ is a set of nodes (or vertices), and $\boldsymbol{E}$ is a set of edges (or arcs) between nodes: $\boldsymbol{E} \subseteq\{(u, v) \mid u, v \in \boldsymbol{N}\}$. We assume that edges are directed, i.e. each edge $(u, v)$ is ordered and it has a head $v$ and a tail $u$. The children $\operatorname{ch}[n]$ of a node $n$ are defined by $\operatorname{ch}[n]=\{u \mid(n, u) \in \boldsymbol{E}\}$. Finally, a graph is called acyclic if there is no path, i.e. a sequence of connected edges, that starts and ends in the same node ${ }^{2}$ otherwise it is cyclic.

\footnotetext{
${ }^{2}$ The considered paths can be of any length and be simple (without repeating nodes) or not.
} 
Fig. 2 In a graph, nodes represent concepts and edges relationships. The state $x_{1}$ is computed by the transition function that uses the label $l_{1}$ of node 1 , the states $\boldsymbol{x}_{2}, \boldsymbol{x}_{4}, \boldsymbol{x}_{6}$ and the labels $l_{2}, l_{4}, l_{6}$ of the children of 1

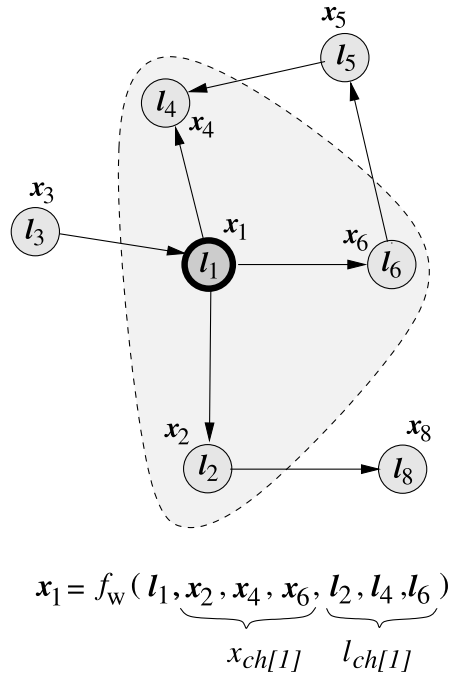

Connectionist models for graph processing assume that the data can be represented as directed graphs, standing for a set of concepts (the nodes) connected by relationships (the edges). The direction of each edge represents the dependence of a concept on another one, i.e. the edge $(n, u)$ denotes the fact that concept $n$ can be defined using concept $u$. Moreover, each node $n$ has a feature vector of real values $\boldsymbol{l}_{n}$, called label, that describes some properties of the concept.

In order to implement this idea, a real vector $\boldsymbol{x}_{n} \in \mathbb{R}^{s}$, called state, is attached to each node $n$ (see Fig. 2). The state should contain a description of the concept represented by the node, so the state of a node naturally depends on its label and on its children. Formally, $\boldsymbol{x}_{n}$ is computed by a parametric function $f_{w_{n}}$, called state transition function, that combines the information attached to node $n$ and to its children $\operatorname{ch}[n]$

$$
\boldsymbol{x}_{n}=f_{\boldsymbol{w}_{n}}\left(\boldsymbol{l}_{n}, \boldsymbol{x}_{\mathrm{ch}[n]}, \boldsymbol{l}_{\mathrm{ch}[n]}\right), \quad n \in \boldsymbol{N},
$$

where $\boldsymbol{x}_{\operatorname{ch}[n]}$ and $\boldsymbol{l}_{\operatorname{ch}[n]}$ are the states and the labels of the nodes in $\operatorname{ch}[n]$, respectively. The transition function $f$ is implemented by a neural network and the parameters $w_{n}$ are the weights of this network. Although the transition function can adopt a different set of parameters for each node, as suggested by the notation $\boldsymbol{w}_{n}$, such a solution is not useful, since it would give rise to a model without generalization capability. In practice, only two solutions are used in the existing models: all the nodes share the same parameters, so that $\boldsymbol{w}_{n}=\boldsymbol{w}$ holds; a set of parameters is shared by a group of nodes and each node $n$ has a type $k_{n}$ that defines the group it belongs to, i.e. $\boldsymbol{w}_{n}=\boldsymbol{w}_{k_{n}}$. For example, in a dataset that represents a relational database, where nodes denote tuples, the type $k_{n}$ is naturally defined by the table the tuple $n$ belongs to. It is worth noticing that if a node type is used, nodes having different type may even use different transition functions and feature vectors, i.e. $l_{n} \in \mathbb{R}^{d_{k_{n}}}$ and $f_{w_{n}}=f_{w_{k_{n}}}^{k_{n}}$ may hold.

Moreover, for each node $n$ an output vector $\boldsymbol{o}_{n}$ is also computed that depends on the state $\boldsymbol{x}_{n}$ and the label $\boldsymbol{l}_{n}$ of the node. The dependence is modeled by a parametric output function $g_{w_{n}}$

$$
\boldsymbol{o}_{n}=g_{w_{n}}\left(\boldsymbol{x}_{n}, \boldsymbol{l}_{n}\right), \quad n \in N .
$$


Together, (1) and (2) define a parametric model that computes an output $\boldsymbol{o}_{n}=\varphi_{\boldsymbol{w}}(\boldsymbol{G}, n)$ for each node $n$ of the graph $\boldsymbol{G}$, taking into account the labels and the relationships of all the nodes in $\boldsymbol{G}$. The parameter set $\boldsymbol{w}$ includes all the $\boldsymbol{w}_{n}$ used by the transition and the output functions. ${ }^{3}$

Graph and relational neural networks are supervised learning models. In the supervised framework, the node output $\varphi_{\boldsymbol{w}}(\boldsymbol{G}, n)$ predicts a property of the concept represented by $n$. Thus, a supervised learning set $\mathcal{L}$ can be defined as a set of triples $\mathcal{L}=\left\{\left(\boldsymbol{G}_{i}, n_{i, j}, \boldsymbol{t}_{n_{i, j}}\right) \mid 1 \leq\right.$ $\left.i \leq p, 1 \leq j \leq q_{i}\right\}$, where each triple $\left(\boldsymbol{G}_{i}, n_{i, j}, \boldsymbol{t}_{n_{i, j}}\right)$ denotes a graph $\boldsymbol{G}_{i}$, one of its nodes $n_{i, j}$ and the desired output $\boldsymbol{t}_{n_{i, j}}$. Moreover, $p$ is the number of graphs in $\mathcal{L}$ and $q_{i}$ is the number of the supervised nodes in graph $\boldsymbol{G}_{i}$, i.e. the nodes for which a desired output is given. The goal of the learning procedure is to adapt the parameters $\boldsymbol{w}$ so that $\varphi_{\boldsymbol{w}}$ approximates the targets of supervised nodes. In practice, the learning problem is often implemented by the minimization of the quadratic error function

$$
e_{\boldsymbol{w}}=\sum_{i=1}^{p} \sum_{j=1}^{q_{i}}\left(\boldsymbol{t}_{n_{i, j}}-\varphi_{\boldsymbol{w}}\left(\boldsymbol{G}_{i}, n_{i, j}\right)\right)^{2} .
$$

As in common feedforward neural networks, several optimization algorithms can be used: almost all of them are based on a subprocedure that computes the error gradient w.r.t. the weights. When the gradient is available, the possible optimization methods include, for instance, gradient descent, scaled conjugate gradient, Levenberg-Marquardt and resilient backpropagation (Haykin 1994).

RelNNs, GNNs and the other neural models for graph processing differ with regard to the implementation of the two functions $f_{\boldsymbol{w}_{n}}$ and $g_{\boldsymbol{w}_{n}}$, and to the method adopted to compute the states $\boldsymbol{x}_{n}$ and the gradient of the error function $\frac{\partial e_{w}}{\partial \boldsymbol{w}}$. Those differences will be described in the following sections.

\subsection{Learning algorithms}

All the models we consider are based on a common idea. The graph processing defined by (1) and (2) can also be described as the computation carried out on a large network, called encoding network, that has the same topology as the input graph. The encoding network is obtained by substituting all the nodes of $\boldsymbol{G}$ with "units" that compute the function $f_{w_{n}}$. The units are connected according to graph topology (Fig. 3), where the directions of the arcs are inverted. The " $f$-units" calculate the states locally at each node. The information is diffused through the encoding network following the connections defined by the edges: while in the input graph edge directions express the dependencies between nodes, in the encoding network they define the direction of the information flow. For the nodes where the output is computed, the " $f$-unit" is also connected to another unit that implements the output function $g_{w_{n}}$.

Actually, as clarified in the following, the encoding network can be used both to compute the states and to adapt the parameters. Since the encoding network connectivity depends on the input graph, in some approaches the input domain is limited in order to simplify

\footnotetext{
${ }^{3}$ Different versions of (1) and (2) can be used, without affecting or even improving the expressive power of the model. For instance, a more general model can process also edge labels by including their codings into the inputs of $f_{w}$, i.e., replacing (1) by $\boldsymbol{x}_{n}=f_{\boldsymbol{w}_{n}}\left(\boldsymbol{l}_{n}, \boldsymbol{x}_{\mathrm{ch}[n]}, \boldsymbol{l}_{\mathrm{ed}[n]}, \boldsymbol{l}_{\mathrm{ch}[n]}\right)$, where $\boldsymbol{l}_{\mathrm{ed}[n]}$ are the labels of the edges coming out from $n$. On the other hand, removing the node label from the input parameters of $g_{w}$ does not affect the expressive power, since such an information is already included in $f_{w}$. In this paper, for sake of simplicity, we describe only the simplest general model that includes both GNNs and RelNNs.
} 

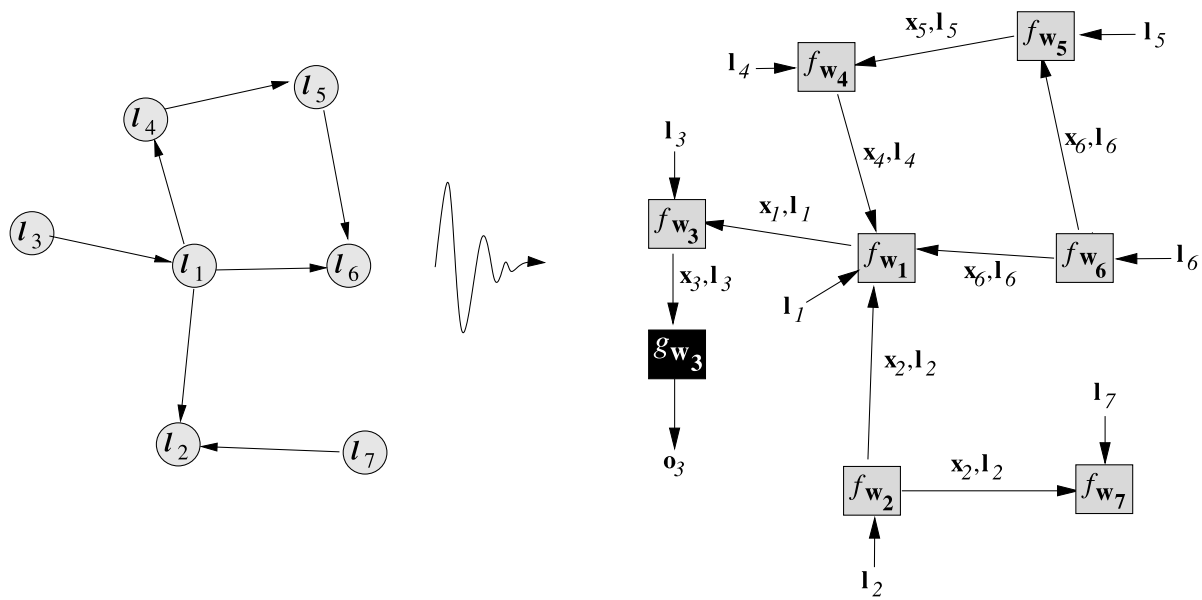

Fig. 3 A graph and the corresponding encoding network. The computation is carried out by " $f$-units" locally at each node and the information is diffused according to the graph connectivity. An output unit computes the output at node 3

the learning and the testing procedures. In fact, the main difference between RelNNs and GNNs is that the former model assumes that the input graph $\boldsymbol{G}$ is acyclic and has a root node. ${ }^{4}$ The root is the only supervised node and RelNNs produce just one output for each graph. Thus, RelNNs can be adopted only on those tasks where the goal is to classify the concept represented by the whole graph, whereas GNNs make no assumption on the input domain and can produce a different output for each node, i.e. they can classify also the single concepts denoted by the nodes.

\subsubsection{Relational neural networks}

If a graph is acyclic, the corresponding encoding network turns out to be a large feedforward neural network whose components are the neural network units implementing $g_{w_{n}}$ and $f_{w_{n}}$ (see Fig. 4). Thus, there is an order in which the units should be updated to propagate the signals from inputs to outputs. Going in the reverse direction, from outputs to inputs, makes it possible to backpropagate the error signal and compute the gradient.

In other words, a common backpropagation procedure (McClelland et al. 1986) can be applied on the encoding network in order to obtain the states $\boldsymbol{x}_{n}$ and the gradients $\frac{\partial e_{w}}{\partial \boldsymbol{w}_{n}}$. More precisely, the states $\boldsymbol{x}_{n}$ are evaluated by the networks $f_{\boldsymbol{w}_{n}}$ following the natural order defined by the edges ${ }^{5}$ : first the states of the nodes without children are calculated, then the states of their parents, and so on until the state of the root is obtained. Finally, the output is produced by $g_{w_{n}}$.

On the other hand, the gradient $\frac{\partial e_{w}}{\partial w}$ is calculated by backpropagating the error from the root to the leaves. More precisely, the derivative of the error with respect to the node state $\frac{\partial e_{w}}{\partial x_{n}}$ is computed first for the root, then for its children, and so on, until the leaves are

\footnotetext{
${ }^{4} \mathrm{~A}$ root node of the graph from which every other node is reachable.

${ }^{5}$ Formally, an edge states that the concept represented by the parent node depends on the concept denoted by the child. Thus, the set of edges define a partial order, called the natural order, that specify the dependencies between the concepts.
} 

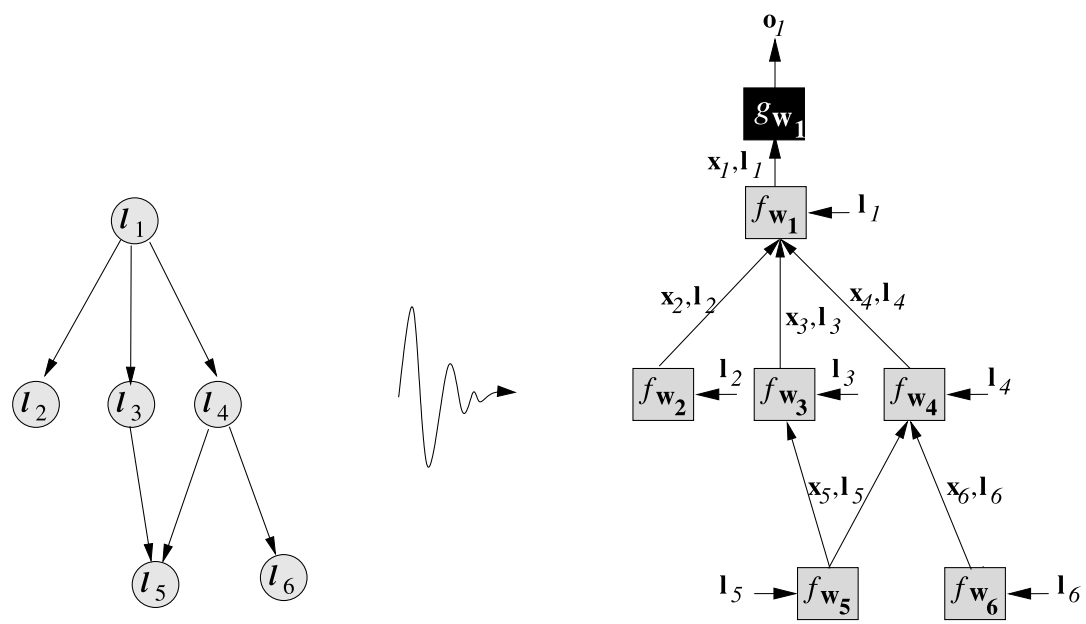

Fig. 4 An acyclic graph and the corresponding encoding network. The backpropagation learning algorithm can be used on the encoding network, which is a feedforward network

reached. For each unit, the value $\frac{\partial e_{w}}{\partial x_{n}}$ allows to calculate the gradient $\frac{\partial e_{w}}{\partial w_{n}}$. Finally, since the parameters are shared among nodes of the same type, the corresponding gradients are accumulated. Such a learning algorithm, which is also used for recursive neural networks, is called backpropagation through structure (Sperduti and Starita 1997; Frasconi et al. 1998).

Moreover, it is worth noting that, even if cyclic graphs cannot be taken in input directly by RelNNs, they can be transformed into trees by an appropriate pre-processing (see Fig. 5 and Algorithm 1). The procedure consists of unfolding the graphs into trees according to a breadth-first visit. The visit starts from the node $n$ where the output is evaluated. At each step, a node $u^{\prime}$ of the unfolding tree $\boldsymbol{T}$ along with a corresponding node $u$ of the graph $\boldsymbol{G}$ are considered: $\boldsymbol{T}$ is extended by connecting $u^{\prime}$ to a new set of children that are exact copies of the children of $u$. The procedure is repeated until $\boldsymbol{T}$ has reached a predefined depth. At the end, $\boldsymbol{T}$ is made up of copies ${ }^{6}$ of the nodes of $\boldsymbol{G}$ such that each copy in $\boldsymbol{T}$ resembles a node in $\boldsymbol{G}$ both for its label and for its local connectivity.

Even if in practical applications the unfolding tree often contains most of the original information, in theory the pre-processing may cause a loss of information, because it may happen that two different graphs and/nodes are unfolded to the same tree. On the other hand, a theoretical condition ensuring that the unfolding is lossless is described in Bianchini et al. (2006), where it is proved that the generated tree contains the same information of the original graph, provided that all the edges are visited and the nodes have distinct labels. See Blockeel and Bruynooghe (2003) for a more detailed description of the unfolding procedure implicit in the RelNN processing scheme.

\subsubsection{Graph neural networks}

The input graph of a GNN, and as a consequence the encoding network, can be cyclic. In this case, backpropagation through structure cannot be used to compute the states and to

\footnotetext{
${ }^{6}$ In general, for each node in $\boldsymbol{G}$ there may exist several copies in $\boldsymbol{T}$. Even if the number of nodes grows exponentially with respect to the tree depth, it is possible to merge the common sub-structures whose dimension is linear with respect to the tree depth, as explained in Sect. 2.1.3.
} 


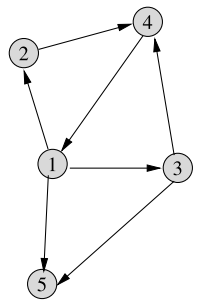

(A)

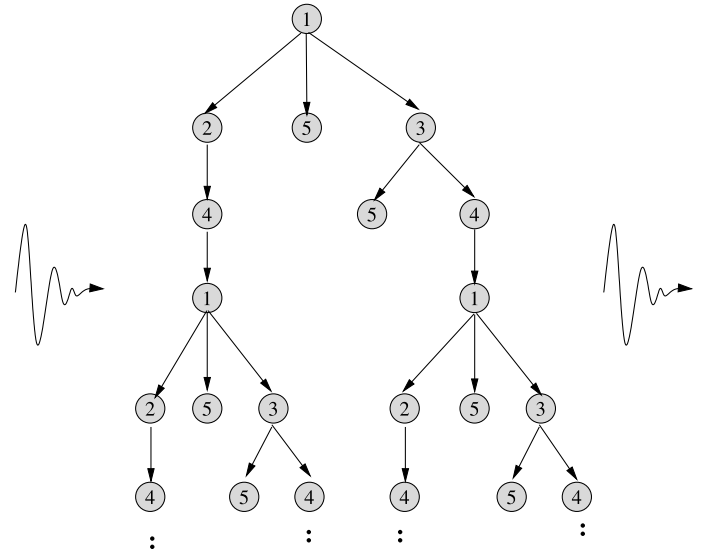

(B)

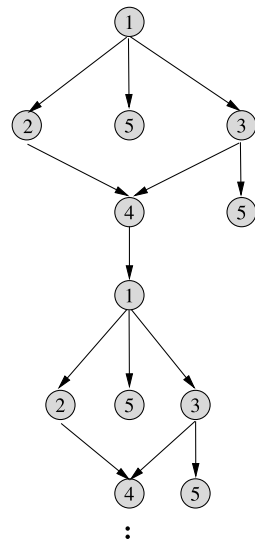

(C)

Fig. 5 A graph (A), its unfolding tree $(\mathbf{B})$ and the corresponding directed acyclic graph (C). The tree (B) is generated visiting the graph $(\mathbf{A})$ with a breadth-first strategy. Graph $(\mathbf{C})$ is generated by merging common sub-trees in (B)

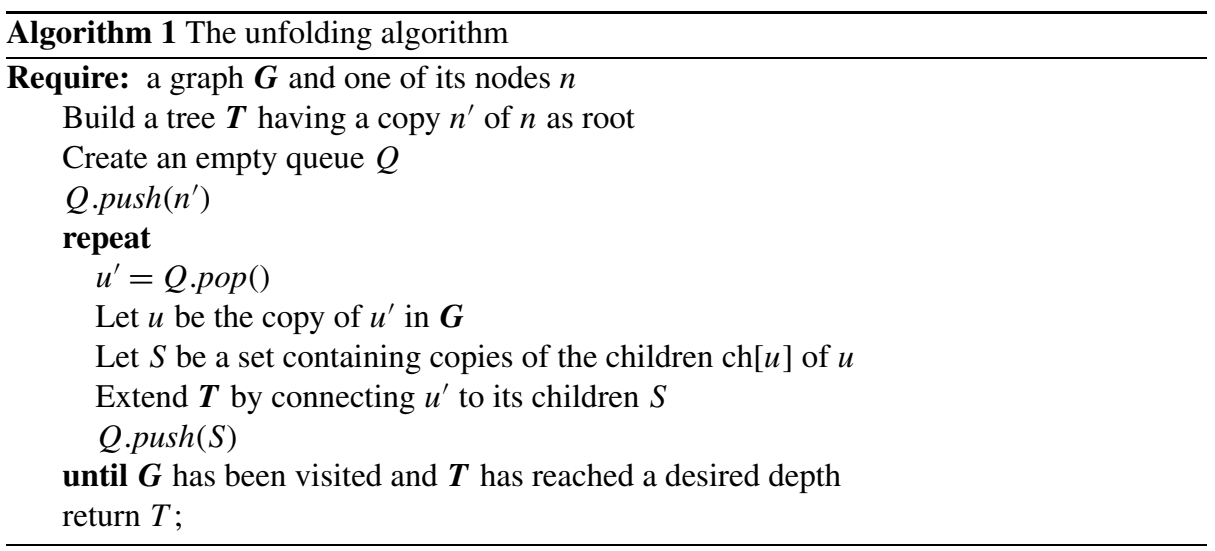

train the network. Thus, three issues have to be addressed in order to implement GNNs: (a) as the states $\boldsymbol{x}_{n}$ are defined recursively, depending one on the other, it must be ensured that they are defined unambiguously; (b) a method must be designed to compute the states $\boldsymbol{x}_{n}$; (c) an algorithm is required to compute the gradient $\frac{\partial e_{w}}{\partial w}$. Those issues are addressed in the following.

- Existence and uniqueness of the states $\boldsymbol{x}_{n}$. Let $\boldsymbol{F}_{\boldsymbol{w}}$ and $\boldsymbol{G}_{\boldsymbol{w}}$ be the vectorial functions obtained by stacking all the instances of $f_{w}$ and $g_{w}$, respectively. Then (1) and (2) can be rewritten for the GNN model as

$$
\boldsymbol{x}=\boldsymbol{F}_{w}(\boldsymbol{x}, \boldsymbol{l}), \quad \boldsymbol{o}=\boldsymbol{G}_{w}(\boldsymbol{x}, \boldsymbol{l}),
$$

where $\boldsymbol{l}$ represents the vector containing all the labels of the input graph and $\boldsymbol{x}$ collects all the states. By the Banach fixed point theorem (Khamsi 2001), if $\boldsymbol{F}_{\boldsymbol{w}}$ is a contraction 
mapping, ${ }^{7}$ then (4) has a unique solution. Thus, issue (a) can be solved by designing $f_{w}$ such that the global function $\boldsymbol{F}_{\boldsymbol{w}}$ results to be a contraction mapping w.r.t. the state $\boldsymbol{x}$. In practice, this goal can be achieved by adding a penalty term $p\left(F_{w}\right)$ to the error function

$$
e_{\boldsymbol{w}}=\sum_{i=1}^{p} \sum_{j=1}^{q_{i}}\left(\boldsymbol{t}_{n_{i, j}}-\varphi_{\boldsymbol{w}}\left(\boldsymbol{G}_{i}, n_{i, j}\right)\right)^{2}+\beta p\left(F_{\boldsymbol{w}}\right)
$$

where $p\left(F_{w}\right)$ measures the contractivity of $F_{w}$ and $\beta$ is a predefined parameter balancing the importance of the penalty term with respect to the error achieved on patterns. ${ }^{8}$ More details can be found in Gori et al. (2005), Scarselli et al. (2009b).

- Computation of the states $\boldsymbol{x}_{n}$. The Banach fixed point theorem also suggests a method to solve issue (b). In fact, the theorem states that if $\boldsymbol{F}_{\boldsymbol{w}}$ is a contraction mapping, then the dynamical system $\boldsymbol{x}(t+1)=\boldsymbol{F}_{\boldsymbol{w}}(\boldsymbol{x}(t))$, where $\boldsymbol{x}(t)$ denotes the proposed $t$-th iterate of $\boldsymbol{x}$, converges exponentially fast to the solution of (4). In other words, the states can be simply computed by an iterative application of their definition, i.e. by the following dynamical system. ${ }^{9}$

$$
\boldsymbol{x}_{n}(t)=f_{\boldsymbol{w}}\left(\boldsymbol{l}_{n}, \boldsymbol{x}_{\mathrm{ch}[n]}(t-1), \boldsymbol{l}_{\mathrm{ch}[n]}\right), \quad n \in N .
$$

Intuitively, each iteration corresponds to an activation of the f-units in the encoding network. The computation is stopped when the state change becomes small, i.e. $\|\boldsymbol{x}(t)-\boldsymbol{x}(t-1)\| \leq \varepsilon$ for a vectorial norm $\|\cdot\|$ and a predefined small real number $\varepsilon>0$.

- Computation of the gradient. In order to design a gradient descent learning algorithm, we can observe that the encoding network represents a system having a settling behavior. For this reason, the gradient can be computed using the Almeida-Pineda algorithm (Almeida 1990; Pineda 1987). In fact, GNNs compute the gradient by a combination of the backpropagation through structure algorithm and the Almeida-Pineda algorithm which consists of two phases:

(a) The states $\boldsymbol{x}_{n}(t)$ are iteratively updated, using (5), until they are close to the fixed point at step $r$

(b) The gradient $\frac{\partial e_{w}}{\partial w}$ is calculated by backpropagating the error signal through the encoding network from step $r$ back to previous steps, until the error signal is close to 0 . Then, the weights are updated.

Thus, while phase (a) moves the system to a stable point, phase (b) adapts the weights to change the outputs towards the desired target. The two phases are iterated until some stop

\footnotetext{
${ }^{7}$ A function $\rho: \mathbb{R}^{n} \rightarrow \mathbb{R}^{n}$ is a contraction mapping w.r.t. a vector norm $\|\cdot\|$, if there exists a real number $\mu, 0 \leq \mu<1$, such that for any $\boldsymbol{x}_{1}, \boldsymbol{x}_{2} \in \mathbb{R}^{n},\left\|\rho\left(\boldsymbol{x}_{1}\right)-\rho\left(\boldsymbol{x}_{2}\right)\right\| \leq \mu\left\|\boldsymbol{x}_{1}-\boldsymbol{x}_{2}\right\|$.

${ }^{8}$ More precisely, the proposed approach cannot ensure that the transition function $F_{w}$ is a contraction mapping outside of the training set. In theory, a GNN may not be able to compute a unique state, if it is applied on a graph which is different from those already observed in training set. However, in practice, such a case would not have any particular consequence except for the wrong prediction produced for the current input graph. More importantly, such a behaviour has never been observed in the experiments. It is also worth mentioning that there exists another version of the GNN model, called linear GNN (Scarselli et al. 2009b), which does not suffer from this limitation and was not used in this paper since, according to previous experiments, its performance is lower.

${ }^{9}$ Since, by Banach theorem, the fixed point is unique, the stable point computed by the dynamical system does not depend on the initial state.
} 
criterion is fulfilled. It is worth to mention that even if the gradient could be computed also by applying the standard backpropagation through time algorithm (Werbos 1990) to the encoding network, however the procedure adopted by GNNs is faster and uses less memory by exploiting Almeida-Pineda algorithm peculiarities. More details can be found in Gori et al. (2005), Scarselli et al. (2009b).

\subsubsection{Computational complexity issues}

The time computational cost of learning in RelNNs is mainly due to the forward and the backward phases of the backpropagation algorithm. The two phases have linear cost with respect to the dimension of the encoding network. When the input graph is acyclic, the encoding network has exactly the same shape of the graph, so that each step of the learning algorithm costs $O(\max (|\boldsymbol{N}|,|\boldsymbol{E}|))$, i.e., the cost is linear with respect to the number of nodes and the number of edges in $\boldsymbol{G}$.

A similar analysis applies to the case when the input graph is cyclic, provided that we consider the unfolding tree of $\boldsymbol{G}$ instead of $\boldsymbol{G}$ itself. One may wonder whether the dimension of unfolding trees is a problem, since the number of nodes grows exponentially with respect to the tree depth. However, unfolding trees can be easily reduced by merging the common sub-structures (see Fig. 5). In fact, two nodes can be fused provided that they have the same label and their children have been already fused. Such a merging procedure can be repeated from leaves to root until, in each level of the tree, there are at most as many nodes as in $\boldsymbol{G}$. The result is an acyclic graph with less than $O(d \cdot|N|)$ nodes, where $d$ is the maximum depth of trees, that contains the same information of the original tree and can be processed by a RelNN.

On the other hand, the GNN learning algorithm requires $O(r \cdot \max (|\boldsymbol{N}|,|\boldsymbol{E}|))$ operations, where $r$ is the number of iterations needed to compute the state by (5). Such a claim is explained observing that both each iteration of (5) and each step of backpropagation costs $O(\max (|\boldsymbol{N}|,|\boldsymbol{E}|))$ (see Scarselli et al. 2009 b for more details). Interestingly, $r$ is usually small due to the fact that the convergence to the fixed point is exponential.

Thus, learning has a similar cost in RelNNs and in GNNs. The difference is mainly due to the values $d$ and $r$, which, are determined in two different ways: in RelNN, $d$ is a predefined parameter of the pre-processing procedure; in GNNs, $r$ is dynamically determined during learning.

\subsection{Transition and output functions}

RelNNs and GNNs differ also for the implementation of the transition and the output functions.

\subsubsection{Relational neural networks}

Relational neural networks have the following peculiarities:

- The output function $g_{w_{n}}$ is implemented by a feedforward neural network.

- The transition function $f_{w_{n}}$, which does not use the labels $\boldsymbol{l}_{n}$, is implemented by a recurrent neural network $r_{w_{n}}$ that combines the states and the labels of the children of each node $n$, storing the result into an internal state $z(i) \in \mathbb{R}^{s}$ (see Fig. 6(A)). Formally,

$$
z(i)=r_{w_{n}}\left(\boldsymbol{x}_{\mathrm{ch}_{i}[n]}, \boldsymbol{l}_{\mathrm{ch}_{i}[n]}, z(i-1)\right)
$$


Fig. 6 The implementation of the transition function in RelNNs (A) and GNNs (B)

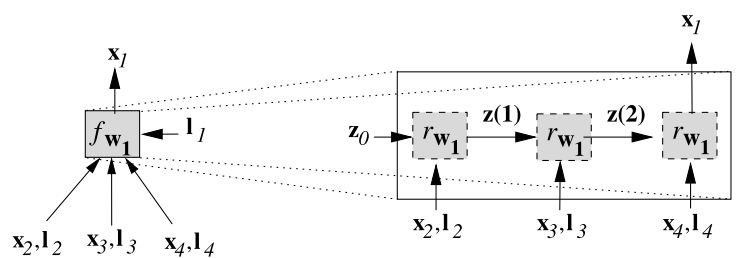

(A)

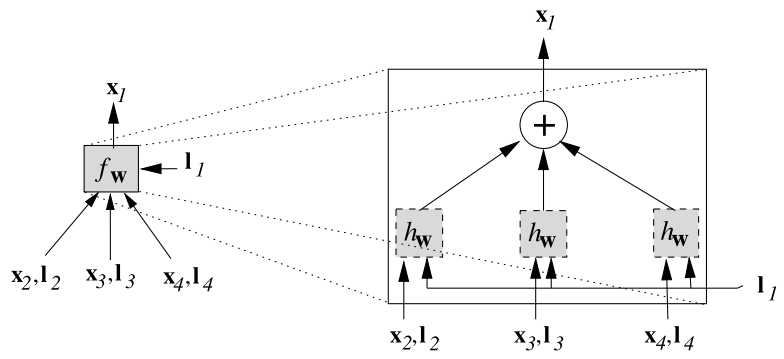

(B)

where $\operatorname{ch}_{i}[n]$ is the $i$-th child of $n$ and $z(0)=z_{0}$ is a default initial value. The recurrent network processes a child at every time step $i$, following some predefined order, which depends on the considered application. The final internal state is then used as the state of the node, i.e., $\boldsymbol{x}_{n}=\boldsymbol{z}(|\operatorname{ch}[n]|)$. Notice that different kinds of recurrent neural networks have been proposed and can be used to implement $r_{w_{n}}$ as, for instance, fully recurrent networks and locally recurrent networks (Back and Tsoi 1994). It is also worth mentioning that the order according to which the children are processed may affect the performance of RelNNs, particularly when such an order is arbitrary and it does not depend on domain knowledge. However, previous experiments have shown that the importance of the issue can be mitigated by shuffling the children during the learning (Uwents and Blockeel 2005).

- Nodes are grouped per type. Different networks are used for different types of nodes. Each network can have a different number of inputs and parameters. The weights are only shared between networks of the same type.

\subsubsection{Graph neural networks}

Graph neural networks have the following characteristics:

- No distinction is made between the nodes in the graph. All the nodes share the same transition function and the same output function, i.e., $f_{w_{n}}=f_{w}, g_{w_{n}}=g_{w}$ and the same parameters $\boldsymbol{w}_{n}=\boldsymbol{w}$.

- In GNNs, the transition function $f_{w}$ is implemented as a sum of contributions (see Fig. 6(B)). Each contribution is related to a child and is produced by a feedforward neural network $h_{w}$ that takes in input the state and the label of the considered child and the label 
of the node ${ }^{10}$

$$
\boldsymbol{x}_{n}=f_{\boldsymbol{w}}\left(\boldsymbol{l}_{n}, \boldsymbol{x}_{\mathrm{ch}[n]}, \boldsymbol{l}_{\mathrm{ch}[n]}\right)=\sum_{i=1}^{|\operatorname{ch}[n]|} h_{\boldsymbol{w}}\left(\boldsymbol{l}_{n}, \boldsymbol{x}_{\mathrm{ch}_{i}[n]}, \boldsymbol{l}_{\mathrm{ch}_{i}[n]}\right) .
$$

Notice that, differently from the RelNN solution, $\boldsymbol{x}_{n}$ does not depend on the order in which the children are processed. Obviously, this latter technique is advantageous or not according whether, in the considered application domain, important information can be encoded by an order relationship between the children. ${ }^{11}$

Interestingly, GNNs are apparently limited by the fact that the global transition function $\boldsymbol{F}_{w}$ has to be a contraction map and that (7) is used in place of (1). On the other hand, GNNs have been proved to be able to approximate in probability any function $\varphi$ on graphs under mild conditions on the input domain and on the set of considered functions. For instance, the universal approximation holds provided that $\varphi$ is continuous with respect to the graph labels and any node of each input graph can be distinguished from the other nodes either by mean of the label (the nodes have different labels) or by mean of the connectivity (the nodes belong to different paths). The result holds for non-positional graphs, when the transition function (7) is adopted, and for positional graphs, when (1) is used. More details can be found in Scarselli et al. (2009a).

\subsection{Related approaches}

In this section, the connectionist approaches for graph processing are briefly reviewed discussing their relationship with the framework of (1) and (2). Larger reviews and other attempts to describe the common features of those approaches can be found in GoulonSigwalt-Abram et al. (2005), Hammer et al. (2004b).

Common recurrent networks can be considered the simplest method to deal with a nonstatic domain, which, in this case, consists of sequences of real vectors. In fact, a sequence can be straightforwardly represented by a graph where the nodes are connected in a line. On the other hand, the actual ancestor of the connectionist methods for graph elaboration is the Recursive Neural Network (RNN) model (Goller and Küchler 1996; Sperduti and Starita 1997; Frasconi et al. 1998). RNNs are similar to RelNNs as they can process acyclic graphs having a root, but in the former approach the transition function $f_{\boldsymbol{w}_{n}}$ is directly implemented by a feedforward neural network, instead of a recurrent network. ${ }^{12}$

The literature contains a number of extensions of the recursive model. For example, different transition and output functions were proposed in Bianchini et al. (2005) in order to

\footnotetext{
${ }^{10}$ Interestingly, both RelNNs and GNNs use specialized versions of $f_{w}$. Actually, $h_{w}$ in (7) and $r_{w}$ in (6) are preferred to the general $f_{w}$ in (1), because they allow to easily deal with application domains where the number of children for each node is highly varying.

${ }^{11}$ Notice that, in theory, the GNN transition function can be also used to process ordered graphs, provided that an extra input, which codifies the position of the child, is added to $h_{w}$. However, as far as we know, such an extension has not been experimentally evaluated, yet.

${ }^{12}$ It is worth noticing that whereas the number of parameters of a feedforward neural network is predefined, the number of inputs of the transition function, which include the states of the children, is different for each node. Thus, the network implementing $f_{\boldsymbol{w}_{n}}$ is usually designed with a predefined number of inputs large enough to be able to process the nodes with the maximal number of children: if the node has a smaller number of children, then the input is appropriately padded. Thus the RNN implementation of the transition function is more suited for those application domains where the number of children of a node is small and has a small variance whereas in other cases the RelNN implementation is preferable.
} 
process graphs with edge labels. The transition function studied in Bianchini et al. (2001) permits to deal with non-positional graphs, where no order relationship is defined between the children of a node. The transition function defined in Micheli et al. (2004) is based on a cascade correlation network, while the architecture in Micheli (2009) is automatically constructed. On the other hand, the graph unfolding procedure, which has been described in Sect. 2.1.1, was previously used in Bianchini et al. (2006) in order to apply recursive neural networks on cyclic graphs.

Finally, some effort has been dedicated to the study of the theoretical properties of RNNs and a sort of universal approximation property was proved. In fact, RNNs can approximate in probability any function on trees (Hammer 1999). Such a property is the counterpart of that proved for GNNs.

The schema of (1) and (2) has also been used to design unsupervised methods. In this case, the network parameters are adapted to obtain a clustering/auto-organization of the concepts represented by the graph nodes. After the training, the output $o_{n}$ is considered a coding of the concept denoted by $n$. In SOM for Structured Domains (SOM-SD) (Hagenbuchner et al. 2003), the output network does not exist, i.e., $\boldsymbol{o}_{n}=\boldsymbol{x}_{n}$ holds, while the transition function is a SOM neural network. The state of the node $x_{n}$ is a codebook that identifies the cluster to which the concept belongs. In Labeling RAAM (LRAAM) (Sperduti 1994), the transition network is based on auto-associators. More precisely, a feedforward neural network $k_{w}$ (the auto-associator) takes in input, for each node $n$, the states of the children and the node label $\left[\boldsymbol{l}_{n}, \boldsymbol{x}_{\mathrm{ch}[n]}\right]$. The auto-associator is trained to reproduce in output a copy of the input, in order to force the auto-associator to produce a coding of the input into the hidden layer: later such a coding is assigned to $\boldsymbol{x}_{n}$. In practice, in LRAAM, the transition function consists of the function implemented by the first layer of the above mentioned auto-associator, whereas the output layer is the identity function.

In LRAAM and SOM-SD, the states were defined each at a time following a predefined order among the nodes. Such an assumption, which corresponds to dealing with directed graphs, has been recently overcome by models, for instance Contextual SOM for Structured Domain (CSOM-SD), that can update each state several times (Hagenbuchner et al. 2005, 2009). Other general unsupervised models can be found in Hammer et al. (2004a, 2004b).

From a practical viewpoint, the above mentioned models mostly differ for the learning framework, supervised or unsupervised, and for the kind of graphs they can process. Thus, in order to select the best model, the application domain must be carefully studied. For example, GNNs and CSOM-SD can deal with cyclic graphs and allow to produce an output for each node, whereas RNNs and SOM-SD can process only directed acyclic graphs and the output is produced only in correspondence of the root. Moreover, also the use of particular transition function can simplify the elaboration of some particular kind of graphs, for instance non-positional graphs (Bianchini et al. 2001). However, in many applications, several models can be used, both since the data can be represented in different ways and because different approaches can be used for the some kind of graphs. In those cases, the best model can be chosen only by an appropriate experimentation.

Supervised connectionist models for graph processing have been used in several application domains, including protein structure prediction (Baldi and Pollastri 2004; Money and Pollastri 2009), QSAR (Micheli et al. 2001), theorem proving (Goller 1997), image classification and object localization in images (Bianchini et al. 2003; Di Massa et al. 2006), language recognition (Rodriguez 2001; Sturt et al. 2003), logo recognition (Francesconi et al. 1998) and web page ranking (Scarselli et al. 2005). Applications for unsupervised methods include XML clustering and classification (Yong et al. 2006; Hagenbuchner et al. 2006), image classification (Wang et al. 2002) and web document clustering (Bloehdorn and Blohm 2006). 
Finally, graph processing by neural networks is related to other approaches where patterns are represented along with their relationships. For example, Markov chain models can emulate processes where the causal connections among events are represented by graphs. A Markov chain corresponds to a GNN where the transition function is linear and the output is a real value and is equal to the state $o_{n}=x_{n}$. Random walk theory, which addresses a particular class of Markov chain models, has been applied with some success to the realization of web page ranking algorithms (Brin and Page 1998; Kleinberg 1999). Recently, some attempts have been made to extend these models with learning capabilities such that a parametric model representing the behavior of the system can be estimated from training examples (Tsoi et al. 2003, 2006; Chang and Cohn 2000).

More generally, several other statistical methods have been proposed which assume that the dataset consists of patterns and relationships between patterns. Those techniques include kernel machines for graphs (Gärtner et al. 2004), random fields (Lafferty et al. 2001), Bayesian networks (Jensen 1996), statistical relational learning (Getoor and Taskar 2007), transductive learning (Vapnik 1998) and semi-supervised approaches for graph processing (Chapelle et al. 2006). A comparison between connectionist models for graphs and other approaches is complex and out of the scope of this paper. Here, it is sufficient to notice that the most obvious advantage of the neural models is in the low computational complexity of the test phase, which can be carried out in linear time both with respect to the data and the model dimension. Moreover, the approximation capabilities of neural models have been widely investigated proving that they behave as sort of universal approximators. On the other hand, kernel machines have the advantage of being able to generalize well even when the training set is particularly small. Finally, random fields, Bayesian networks and statistical relational learning provide classification mechanisms with a strong foundation on statistical theory, while transductive and semi-supervised approaches allow to easily exploit data without targets.

\section{Experimental results}

In order to evaluate the GNN and the RelNN models on relational data, we tested them on benchmarks that are often used to compare Inductive Logic Programming (ILP) and Machine Learning (ML) techniques. The considered problems include the task of modeling the tables produced by aggregate function queries and two benchmarks dealing with two QSAR problems, i.e., the prediction of the mutagenicity and the biodegradability properties of some molecules. The experiments on the first benchmark are mostly dedicated to evaluate the properties of the two models, while the tests on the other datasets aim at a comparison with other approaches.

The following statements hold for all the experiments. The datasets were split into a training set, a validation set and a test set. Each model was trained for a predefined number of epochs ${ }^{13}$ on the training set and, every 20 epochs, was evaluated on the validation set. The network achieving the best error on the validation set was evaluated also on the test set. The learning procedures of RelNNs and GNNs exploited the resilient backpropagation algorithm (Riedmiller and Braun 1993) to update the network weights on the basis of the gradient. In all the experiments, the resilient algorithm was configured using the default

\footnotetext{
${ }^{13} \mathrm{An}$ epoch is a single step of the learning procedure and it consists of the presentation of the entire training set to the network.
} 
parameters specified in the original paper, while the parameters of the transition and the output networks were randomly initialized in the range [-0.01, 0.01].

In RelNNs, where a recurrent network is used to implement the transition function, the children are processed following an order that is shuffled at each epoch (Blockeel and Bruynooghe 2003; Uwents and Blockeel 2005) during learning, while in testing the order is the same of the original dataset. Moreover, in the problems where only a node has to be supervised, the chosen node is the first of the original dataset. ${ }^{14}$

The GNN simulator adopted is implemented in MATLAB and is available to download at Monfardini and Scarselli (2004), whereas the RelNN simulator is implemented in C.

\subsection{Aggregation functions}

Modeling an aggregate function can be considered the minimal requirement for a relational learner. An aggregate function is applied to a set or bag of tuples and produces a value summarizing a property of the bag content. There are a number of ways to graphically represent a bag of tuples, which involves only two kinds of concepts: the tuple and the set. For example, in a representation (Fig. 7(a)), a node denotes the bag and other nodes stand for the tuples. Bags and tuples are connected by edges, directed from the former to the latter, that indicate the "is-made-of" relationship. The supervised nodes, which ideally contain the field to predict, are those corresponding to the bags. For its simplicity, the task of modeling an aggregate function is well suited to evaluate the basic characteristics of RelNNs and GNNs.

Here, an artificial dataset was exploited. Each bag included from 5 to 10 tuples, while each tuple had 5 random real fields in the interval $[-0.8,0.8]$. The following aggregate functions have been considered: count, sum, maximum, average and median. Except for the count function, which simply computes the number of tuples in the bag, the other aggregate functions are applied only on one attribute of each tuple (the first one). The presence of useless attributes makes more difficult the task of the relational learner, which has to single out the useful data while it is capturing the target function.

For each bag, its dimension was defined by a random integer number generator using a uniform probability distribution in the range $[5,10]$. In order to avoid biases in the results, the construction of the tuples consisted in two steps: first, the aggregation result was defined by a uniform probability distribution; then, a set of tuples that produced or approximated the expected result was generated by a pseudo-random procedure. Such a procedure depended on the particular aggregation function for the production of the first field, whereas the useless fields were always assigned random values uniformly distributed in $[-0.8,0.8]$. Thus, for the maximum function, the first field of each tuple contained random values smaller than the defined maximum, except for one tuple, which was forced to contain the exact result. For the sum, the tuples were recursively generated and, at each step, the first field was assigned a random value smaller than the difference between the current sum of the bag and the expected result; the last tuple was set so that the sum of the bag is the expected one. For the average, the first field was generated using a uniform distribution centered around the fixed

\footnotetext{
${ }^{14}$ It is worth mentioning that the selection of the supervised node and the children ordering can affect the performance: usually such a choice is based on domain knowledge. For example, in image classification, where the nodes can represent homogeneous regions of the image, the node of the central region of the image or the larger region is often chosen for the supervision (Di Massa et al. 2006). For the considered experiments, we could not individuate a piece of domain knowledge that can help in selecting a set of preferable nodes for supervision or a particular ordering of the children. For this reason, we simply chose the first node and the ordering provided in the original datasets.
} 


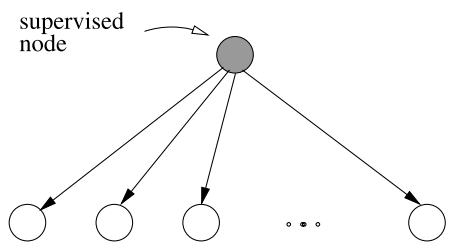

(a)

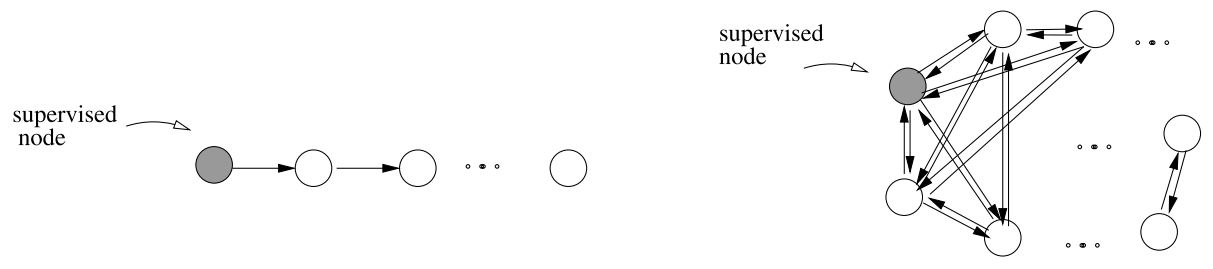

(c)

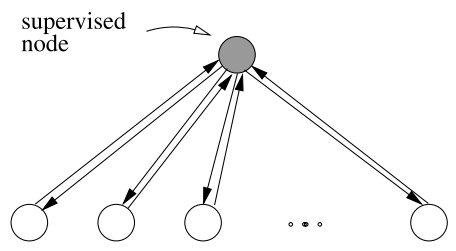

(b)

(d)

Fig. 7 Four graphical representations of a bag of tuples. In (a) and (b), a node stands for the bag and the other nodes denote the tuples. There may be edges directed from the bag to the tuples (a) but also edges in the opposite direction may be used (b). In (c) and (d), the bag is not represented and the tuples are arranged in a sequence (c) or all connected (d)

average. The median dataset was generated in a similar way, but with the median instead of the average.

Each bag was considered correctly predicted if the output of the network was within \pm 0.1 with respect to the target. Results have been averaged over three runs for each function. In each run, the dataset contained 500 bags: 300 in the training set, 100 in validation set and 100 in test set. Moreover, the number of epochs was 1000 .

The experimentation consisted of two parts. In an initial experiment, two basic RelNN and GNN models were tested; then several variations of those models were evaluated to measure the effect on the performance of the model parameters and of the data representation. The configuration of the basic models is:

- State dimension is 5 for both GNNs and RelNNs;

- The GNN transition function $h_{w}$ and the GNN and RelNN output function $g_{w}$ are networks with one hidden layer, 5 hidden neurons, hyperbolic tangent activation function in the hidden neurons and linear activation function in the output neurons;

- In RelNN the transition function is implemented by a recurrent network $r_{w}$ with 5 locally recurrent neurons using the hyperbolic tangent activation function;

- The weights are shared among all the nodes both in GNNs and in RelNNs; ${ }^{15}$

\footnotetext{
${ }^{15}$ Notice that even if RelNNs have the capability to use different networks for different types of nodes in representation (a) such a capability is not useful. In fact, the output function is evaluated only on the compound nodes and the transition functions only on the nodes representing the tuples.
} 
Table 1 The accuracies (percentage) achieved with GNNs and RelNNs on the aggregation function experiment using the base configuration. The sample standard deviation is reported in square brackets

\begin{tabular}{|c|c|c|c|c|c|c|}
\hline \multirow[t]{2}{*}{ Model } & \multicolumn{2}{|l|}{ Count } & \multicolumn{2}{|l|}{ Sum } & \multicolumn{2}{|l|}{$\operatorname{Max}$} \\
\hline & Test & Train & Test & Train & Test & Train \\
\hline GNN & $100[0]$ & $100[0]$ & $100[0]$ & $100[0]$ & 48.3 [9.29] & 61.3 [12.2] \\
\hline RelNN & $99.0[0.23]$ & 99.5 [0.19] & 99.8 [0.09] & $99.9[0.14]$ & 45.1 [3.74] & 63.0 [1.43] \\
\hline \multirow[t]{6}{*}{ Baseline } & 16.7 & 16.7 & 16.0 & 16.0 & 10.8 & 11.0 \\
\hline & \multirow[t]{2}{*}{ Model } & \multicolumn{2}{|l|}{ Avg } & \multicolumn{3}{|l|}{ Median } \\
\hline & & Test & Train & Test & Train & \\
\hline & GNN & $100[0]$ & 99.8 [0.19] & $84.3[0.58]$ & 87.3[ & \\
\hline & RelNN & 99.0 [0.57] & $99.7[0.26]$ & 80.9 [1.86] & 89.32 & 92] \\
\hline & Baseline & 18.4 & 19.0 & 31.0 & 31.2 & \\
\hline
\end{tabular}

- Bags of tuples are represented as in Fig. 7(a). ${ }^{16}$

This configuration was chosen, by a preliminary experimentation, among those achieving the best performances and allowing a simple comparison of the models. However, the purpose was not that of measuring the maximal performance, so that an exhaustive experimentation was not carried out.

Table 1 shows the results obtained by GNNs and RelNNs in the base experiment. Notice that the performance achieved by the two connectionist models varies largely according to the considered aggregation function. This variance partially depends on the general difficulty of neural networks to approximate some functions. For example, it is well know that even a simple feedforward neural network can approximate more easily a function that counts or sums the input values, than a function that computes the maximum. Actually, feedforward neural networks having just one hidden neuron can approximate up to any degree of precision the sum and the count maps (Gori et al. 1998). On the other hand, the approximation of the maximum function requires a number of hidden units that depends on the desired precision. Similarly, the approximation of the average and the median is more difficult because they are composite functions: intuitively, the average requires to sum and to count the tuples, while the median needs to sort and count the tuples.

On the other hand, the performance of the ReINN and the GNN models are very close on all the tasks. Such a fact is probably due to the simplicity of the considered problems and the simplicity of the data representation that does not highlight differences.

GNN and RelNN have been also compared with a baseline approach in last row of Table 1 . The baseline results were obtained by computing an optimal constant output on training set and using such a value as a response to every query. Such a comparison confirms that the two connectionist models learn to combine the information contained in the bags.

More experiments have been carried out in order to evaluate how simple variations to the base configuration affect GNN performance. The first experiment compared different representations of a bag of tuples. More precisely, those depicted in Fig. 7 were considered. Representation (b) is equal to (a) with the difference that also the "belongs-to" relationship is used, i.e., there are edges going from the tuples to the sets. In (c), the tuples are arranged

\footnotetext{
${ }^{16}$ It is worth mentioning that RelNNs can directly process such a representation without a preliminary unfolding, since the graph in Fig. 7(a) is a tree.
} 
Table 2 The performance of the GNN model on the aggregation function benchmarks. The first row displays the result of the base configuration, while other rows show the performance of a number of variations: in rows 2-4, different representations of the bags of tuples; in row 5, the label of the parent is not used in transition function. Computation times (CPU elapsed times) are in seconds for each run (averaged on the five different aggregation functions) on a PC with a CPU Athlon 4600+. The average sample standard deviation is reported in square brackets

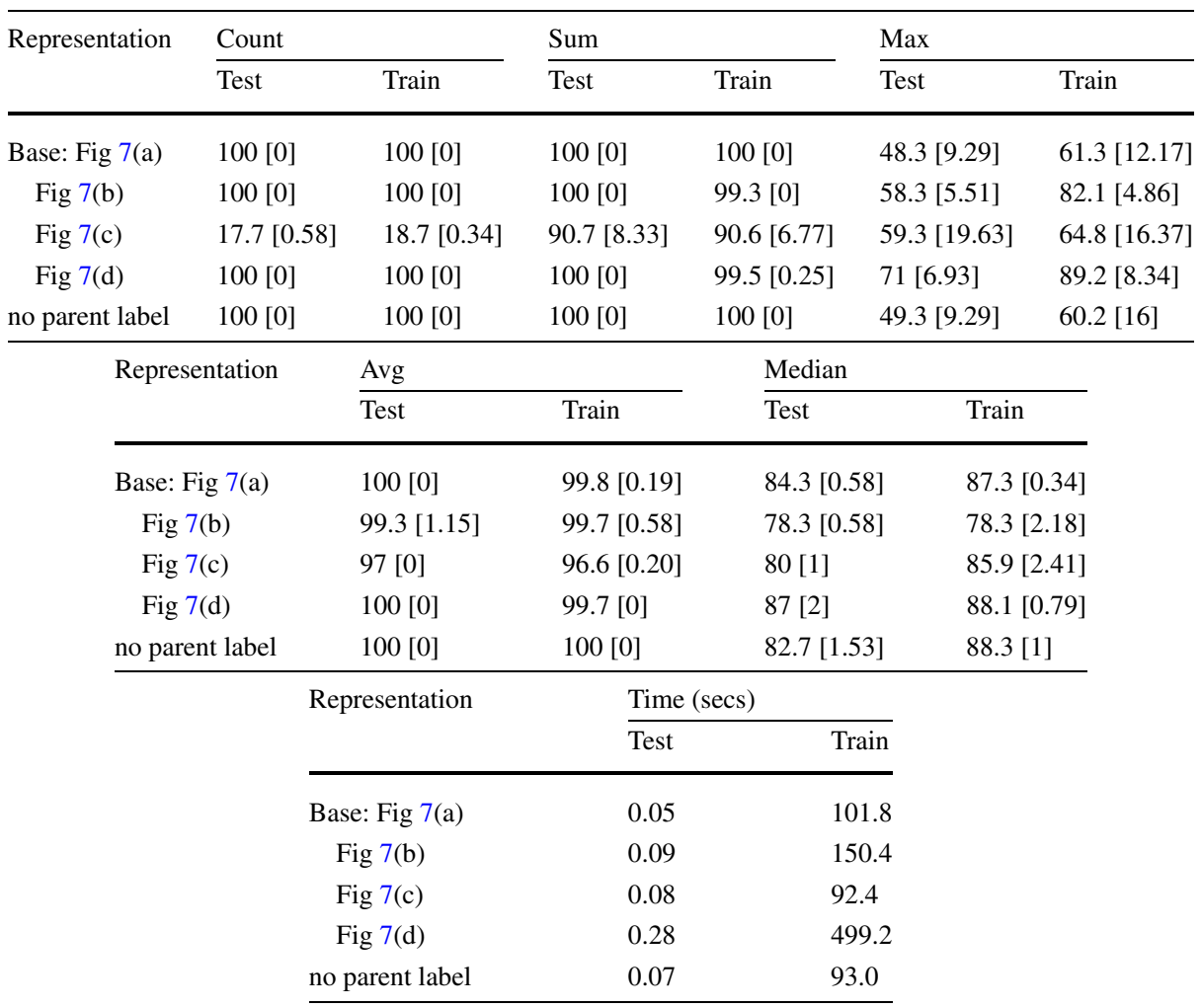

in a sequence where the edges link a tuple to the following one. Finally, in (d) the bag is not represented and all the tuples of a set are connected to each other by edges denoting the "belongs-to-the-same-bag" relationship. Both in (c) and (d), a tuple must be chosen to function as the supervised node: in our experiments, the selected tuple was the first that had been generated, randomly, during dataset creation. Moreover, also the ordering of the tuples in (c) is the one in which they were generated.

Table 2 shows the achieved performances. Interestingly, the best result is obtained with representation (d), while one of the worst results is got by (c). Representation (d) is the one in which the graph diameter ${ }^{17}$ is minimal, whereas representation (c) has the maximal diameter. Thus, the difference in the performance is probably due to the long-term dependencies problem that afflicts also common recurrent networks (Bengio et al. 1994). Intuitively, if the diameter of the graph is large, the encoding network behaves as a deep network and learning

\footnotetext{
${ }^{17}$ The diameter of a graph is the maximal distance between two nodes, where the distance is defined as the minimal number of edges contained in path connecting the nodes.
} 
is difficult, since the derivatives of error w.r.t. the weights rapidly decrease when they are backpropagated.

One may wonder whether the diameter of any input graph could be decreased by adding more edges. However, the edges cannot be chosen disregarding the fact that they have to represent useful information in the considered application domain. Moreover, the computation time is affected by the number of edges in the representation as confirmed by last column of Table 2.

A difference between the standard versions of RelNNs and GNNs is in the information used by the transition networks: the label of the parent is adopted only by GNNs (compare (6) with (7)). This fact may be an advantage, because more information is used, or a disadvantage, because more parameters are needed. In another experiment, the label of the parent node was removed from the transition function commonly used by GNNs, i.e., $h_{\boldsymbol{w}}\left(\boldsymbol{l}_{n}, \boldsymbol{x}_{\mathrm{ch}_{i}[n]}, \boldsymbol{l}_{\mathrm{ch}_{i}[n]}\right)$ in (7) is replaced by $h_{\boldsymbol{w}}\left(\boldsymbol{x}_{\mathrm{ch}_{i}[n]}, \boldsymbol{l}_{\mathrm{ch}_{i}[n]}\right)$. However, the results on the base representation of Fig 7(a) do not single out a clear difference on the performance (see fifth row of Table 2), suggesting that here the two mentioned effects are balanced.

Another set of experiments were dedicated to RelNNs, where the implementation of the transition function is varied. More precisely, three transition networks were evaluated: a locally recurrent neural network (the base configuration), a fully recurrent neural network and the sum of the outputs of a non-recurrent feedforward network adopting the GNN solution described by (7). Fully recurrent neural networks have two layers: the input layer is fully connected to the output one, while the output neurons are also back connected to the input neurons. In locally recurrent networks, there is no feedback from output to input, but there is a back connection from each output neuron to the neuron itself. See Back and Tsoi (1994) for more details on those recurrent models. The parameters of the exploited network were those defined in the basic configuration, i.e., 5 neurons in the output layer, state dimension is 5 , and 5 hidden neurons in the feedforward neural network.

Table 3 shows that the best performance is achieved by the GNN "sum" transition function. In order to explain such a result, it is worth mentioning that, in theory, recurrent networks are a more general model that can implement transition functions which cannot be implemented by combining the outputs of a feedforward network. ${ }^{18}$ However, recurrent networks rely on the order by which the inputs (the children, in this case) are processed. Moreover, recurrent networks are affected by the long-term dependencies problem (Bengio et al. 1994) that limits the performance on long sequences. Thus, when the number of children is large and/or the order is random and does not codify domain information, as in the current experiment, the "sum" transition function can be advantageous over the recurrent networks.

\subsection{The mutagenesis dataset}

The mutagenesis dataset (Debnath et al. 1991) is a small dataset, publicly available (e.g. in Mutagenesis 1991) and often used as a benchmark in the ILP literature (Lodhi and Muggleton 2005). It contains the descriptions of 230 nitroaromatic compounds that are common intermediate subproducts of many industrial chemical reactions. The goal of the benchmark consists of predicting which compounds are mutagenic on Salmonella typhimurium. In the original dataset, the value to be predicted was a real valued measure of the mutagenicity of each compound. In fact, in Debnath et al. (1991) it is showed that 188 molecules out

\footnotetext{
${ }^{18}$ Fully recurrent networks have been proved to be universal approximators on sequences.
} 
Table 3 The performance of the RelNN model on the aggregation function benchmarks. The results achieved by three different kind of transition functions are shown. Computation times (CPU elapsed times) are in seconds. Experiments were conducted on an Intel Core Duo E6850 CPU at $3 \mathrm{GHz}$

\begin{tabular}{|c|c|c|c|c|c|c|}
\hline \multirow[t]{2}{*}{ Representation } & \multicolumn{2}{|l|}{ Count } & \multicolumn{2}{|l|}{ Sum } & \multicolumn{2}{|l|}{$\operatorname{Max}$} \\
\hline & Test & Train & Test & Train & Test & Train \\
\hline \multirow{3}{*}{$\begin{array}{l}\text { Base: locally recurrent } \\
\text { fully recurrent } \\
\text { sum }\end{array}$} & \multirow{3}{*}{$\begin{array}{l}99.0[0.23] \\
99.0[0.35] \\
99.9[0.09]\end{array}$} & 99.5 [0.19] & 99.8 [0.09] & 99.9 [0.14] & $45.08[3.74]$ & 63.0 [1.43] \\
\hline & & $99.2[0.14]$ & 99.7 [0.30] & 99.8 [0.13] & 57.8 [7.24] & 72.2 [4.60] \\
\hline & & $100[0]$ & $99.8[0.14]$ & 99.8 [0.08] & 78.1 [3.00] & 84.1 [3.23] \\
\hline \multirow{2}{*}{\multicolumn{2}{|c|}{ Representation }} & \multicolumn{2}{|l|}{ Avg } & \multicolumn{2}{|l|}{ Median } & \\
\hline & & Test & Train & Test & \multicolumn{2}{|l|}{ Train } \\
\hline \multirow{3}{*}{\multicolumn{2}{|c|}{$\begin{array}{l}\text { Base: locally recurrent } \\
\text { fully recurrent } \\
\text { sum }\end{array}$}} & $99.0[0.57]$ & $99.7[0.26]$ & 80.9 [1.86] & \multicolumn{2}{|c|}{$89.3[0.92]$} \\
\hline & & 96.0 [1.37] & $98.0[0.35]$ & 75.7 [2.29] & \multicolumn{2}{|c|}{$88.6[0.40]$} \\
\hline & & $99.8[0.17]$ & $99.9[0.04]$ & $86.4[1.22]$ & \multicolumn{2}{|c|}{$94.2[0.64]$} \\
\hline & \multirow{2}{*}{\multicolumn{2}{|c|}{ Representation }} & \multicolumn{2}{|c|}{ Time (secs) } & & \\
\hline & & & Test & Train & & \\
\hline & \multirow{2}{*}{\multicolumn{2}{|c|}{$\begin{array}{l}\text { Base: locally recurren } \\
\text { fully recurrent }\end{array}$}} & 0.1 & 34.7 & & \\
\hline & & & 0.1 & 37.4 & & \\
\hline & \multicolumn{2}{|l|}{ sum } & 0.1 & 36.0 & & \\
\hline
\end{tabular}

of 230 are amenable to a regression analysis. This subset was therefore called "regressionfriendly", while the remaining 42 compounds were termed "regression-unfriendly". However, as far as we know, the application considered in all the published papers is a classification problem where it has to be predicted whether a compound is mutagenic (mutagenicity is larger than one) or not (mutagenicity is smaller than one).

In this paper, we concentrate on the classification problem. GNNs and RelNNs were trained to output 1 when they are fed on a mutagenic compound and -1 , when the pattern is not mutagenic. In the testing phase, a compound is predicted to be mutagenic or not according whether the model output is larger than 0 or not.

Despite the fact that the dataset is quite small, it has been used intensively in the past ten year to evaluate statistical and relational learning techniques. For historical reasons, many authors have reported their results only on the "regression-friendly" part, that is often referred to as "the" mutagenesis dataset. Moreover, the comparison is complicated by the fact that many different features can be used in the prediction.

Each compound is provided with four global features (Debnath et al. 1991): two features are chemical measurements (C), namely LUMO, or lowest unoccupied molecule orbital and $\log \mathrm{P}$, or water/octanol partition coefficient, ${ }^{19}$ while the other two features are precoded structural attributes (PS). Moreover, some features describe properties of the single atoms: they include the atom type and the charge. The atom-bond structure is also given, which defines binary relationships between the atoms of each compound. Finally, the presence of functional groups (FG), e.g. methyl groups, have been used in some papers

\footnotetext{
${ }^{19}$ Octanol is a fatty alcohol with eight carbon atoms that is immiscible with water. Water/octanol partitioning, measured in logarithmic scale $(\log \mathrm{P})$, is a relatively good approximation of the partitioning between the cytosol and lipid membranes of living systems.
} 
as higher level features. This last kind of features describes some properties of groups of atoms.

In our experiments, the simplest representation, denoted by $\mathrm{AB}$, includes the atom-bonds and the features of single atoms: the atom type and the charge. Moreover, atom types were represented by a one-hot coding ${ }^{20}$ of the 9 different types available in the dataset. All other features were denoted by the corresponding numerical values: the charge is a real and both $\mathrm{C}$ and PS are 2-dimensional vectors.

The purpose of the experimentation on the mutagenesis dataset and, in the next section, on the biodegradability dataset is to compare, on well known benchmarks from the relational learning field, the performances of RelNNs and GNNs to each other and with respect to other models. Due to the large number of possible choices either in the representation of the data and in definition of the model parameters, an exhaustive comparison of all the possible solutions was not viable. Thus, in the following, we present the results obtained with a configuration that was chosen according both to a preliminary experimentation and some theoretical considerations. Such a preliminary study allowed us to define the most promising configurations for RelNNs and GNNs and the range of the parameters to be experimented.

Two different graphical representations have been considered, that correspond to the cases where the data is represented by two tables (Compounds and Atoms) and one table only (Atoms), see Fig. 8.

(a) Each compound is denoted by a node that is labeled with the global features and is connected to other nodes representing the atoms that belong to the compound. The atom nodes are labeled with the type of the atom and are connected by edges to other nodes

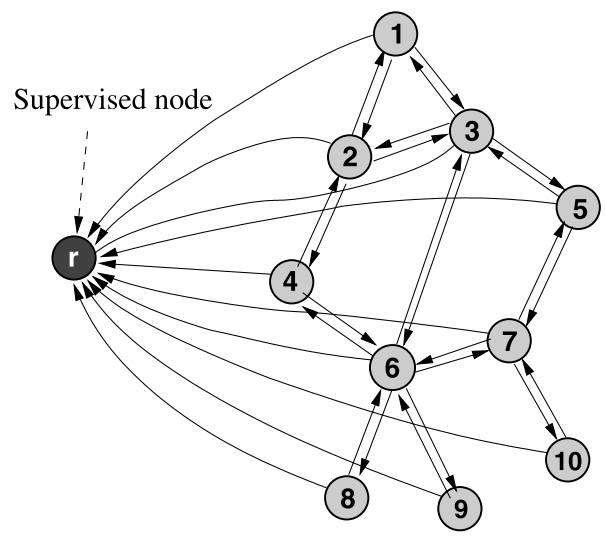

(a)

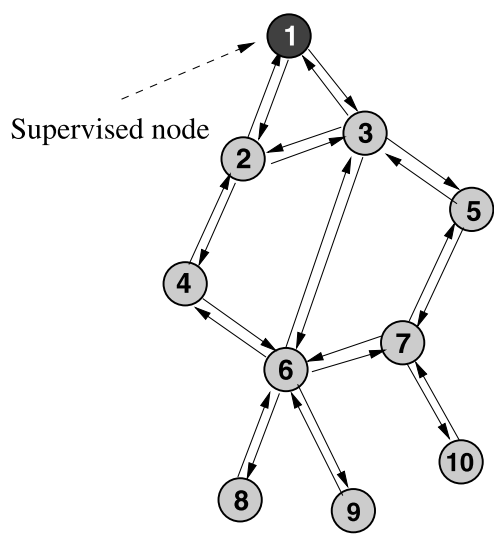

(b)

Fig. 8 The graphical representations for the molecules of the mutagenesis dataset used for RelNNs (a) and for GNNs (b). In (a) the supervised node is a node representing the compound. In (b), the supervised node is one of the nodes representing the atoms

${ }^{20} \mathrm{~A}$ one-hot coding of a variable $v$ that can assume a finite number of different values $v_{1}, \ldots, v_{a}$ consists of a $a$-dimensional vector $\left[t_{1}, \ldots, t_{a}\right]$, such that if $v=v_{i}$, then $t_{i}=1$ and $t_{j}=-1$, for any $j \neq 0$, hold. 


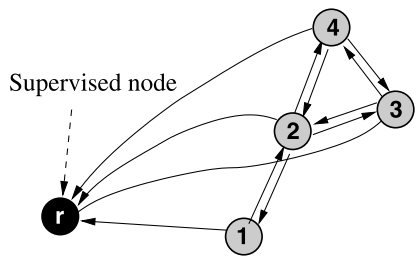

(a)

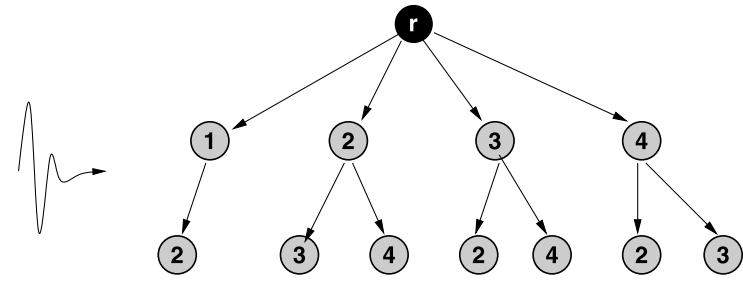

(b)

Fig. 9 The unfolding tree (b) obtained by unfolding the compound (a) up to depth 3. For the sake of clarity, common substructures have not been merged

representing the bonded atoms. The attribute "is_mutagenic", which has to be predicted, is naturally associated with the compound node.

(b) The compound is not represented. The nodes standing for atoms are connected as in (a), while their labels are extended with the global features. The supervised node can be any node: in practice, only the first node ${ }^{21}$ of each compound is supervised.

The experimentation has been carried out using (a) for RelNNs and (b) for GNNs. Actually, the former representation is the more suited to represent the data for RelNNs, whereas the latter is suitable for GNNs. This difference is due to the fact that RelNNs use different neural networks for different relations, while GNNs do not. For this reason, RelNNs can gain an advantage from having two different relations while GNNs cannot. Some preliminary experimental results confirmed that RelNNs achieve the better performance with representation (a), whereas GNNs obtain the better performance with representation (b).

Since representation (a) is cyclic, the graphs must be pre-processed using the unfolding procedure described in Algorithm 1. Figure 9 shows an example of the results of the unfolding of a compound.

Model configuration was as follows.

- The GNN transition function $h_{w}$ and the output function $g_{w}$ were implemented by feedforward neural networks with one hidden layer, hyperbolic tangent activation function in the hidden neurons and linear activation function in the output neurons.

- In RelNNs, the transition function is implemented by a locally recurrent neural network $r_{w_{n}}$ using hyperbolic tangent activations.

Following the experimental procedure commonly adopted on this benchmark, we used a 10 -fold cross-validation scheme. The dataset $\mathcal{L}$ was randomly split into 10 folds $\mathcal{L}_{1}, \ldots, \mathcal{L}_{10}$. For each $i$, an experiment was run using $\mathcal{L} \backslash \mathcal{L}_{i}$ for training and $\mathcal{L}_{i}$ for testing. More precisely, $\mathcal{L} \backslash \mathcal{L}_{i}$ was further randomly split into an training set and a validation set, where the validation set dimension equals the dimension of the test set, i.e., a $10 \%$ of the original dataset The results were averaged on all the folds and on 3 different runs.

Validation sets were used to select the GNN and the RelNN parameter dimensions, i.e. the number of hidden neurons and the state dimension. Nine different configurations were evaluated by testing all the architectures that can be constructed by taking the number of

\footnotetext{
${ }^{21}$ More precisely, the first node is the one corresponding to the first atom in the list of the original dataset. As far we know, the ordering in the dataset has no particular meaning.
} 
hiddens $^{22}$ in 2, 5, 10 and the state dimension in 2, 5, 10. Moreover, the RelNN model has been tested varying also the unfolding depth (in 1,2,3) and the transition function (choosing among a locally recurrent network (lrc), a fully recurrent network (frc) or the sum of the outputs of a feedforward network (sum)). The model architecture achieving the best result on the validation sets was evaluated on test sets. More precisely, the best model is the one obtaining the lower error average over all the folds and all the 3 repetitions. ${ }^{23}$

The number of training epochs was chosen by two different criteria: (a) during training, the considered model was evaluated every 20 epochs on validation set and the epoch corresponding to best performance on validation set was considered the last epoch; (b) a large number of epochs (500 in this case) was chosen, where "large" is heuristically defined as a number several times larger than the number of epochs usually required by the learning algorithm to reach a point where the error does not decreases significantly on training set and on the validation set. ${ }^{24}$ In general, strategy (a) is preferable when a large validation set is available and it provides a precise prediction of the error on test set. On the other hand, the strategy (b) may be better provided that a too long learning time does not cause a loss of generalization capability due to the overfitting phenomenon.

Table 4 shows the performances of GNNs and RelNNs on the two parts of the mutagenesis dataset and on the whole benchmark. The results indicate that, in our experimental setting, stopping criterion (b) (column "500 epochs") is better than criterion (a) (column "Best on val.") for GNNs, whereas the converse holds for RelNNs. In fact, in this case both the validation and the training sets are probably too small: in GNN this gives rise to an early stop of the learning, whereas in RelNN an overfitting phenomenon is observed. ${ }^{25}$ The different behaviour of the two models with respect to overfitting can be confirmed by observing the difference between the performances on training set and on test set, which is small for GNNs and large for RelNNs (see Table 5). The reason of the overfitting in RelNN, which has been not observed on the other datasets of this paper, has not been further investigated, even if it is probably due to the number of parameters, which is larger than in GNN, and to the (eventual) use of recurrent networks, whose performance depends on the sorting of children.

Different sets of features for the labels were tested. More precisely, three cases were considered: only local properties and atom-bonds (denoted by $\mathrm{AB}),{ }^{26} \mathrm{AB}$ and chemical measurements (denoted by $\mathrm{AB}+\mathrm{C}$ ); $\mathrm{AB}+\mathrm{C}$ and structural attributes (denoted by $\mathrm{AB}+\mathrm{C}+\mathrm{PS}){ }^{27}$

\footnotetext{
${ }^{22}$ In order to keep small the number of experiments, only networks with the same number of hiddens in the output function and the transition function were evaluated.

${ }^{23}$ It can be observed that such a procedure introduces a bias in the experiments, since the patterns of a validation set are used also in test sets. On the other hand, the results aggregated by model allow to compare the different configurations.

${ }^{24}$ It is worth to mention that for each experiment only one learning session is run for both the strategies and that, in order to obtain a more fair comparison, the patterns originally assumed to the validation set has not been used to extend the training set in strategy (b).

${ }^{25}$ It is worth to mention that the dimensions of the training set and the validation set have been selected by heuristics and they have not been optimized. Probably, using different sizes for GNNs and RelNNs the performance can be improved. Also, by leave-one-out validation, we could enlarge the training set. However, those solutions have not been considered due to the long time required for running those experiments.

${ }^{26}$ Atom-bonds define the connectivity between the atoms and are not explicitly stored in labels.

${ }^{27}$ Notice that the actual label content depends also on the representation. In representation (b), the labels contain both the local and the global features (C, PS), whereas in representation (a), the local features are stored in the labels of the nodes standing for the atoms and the global features are stored in the labels of the nodes denoting the compounds.
} 
Table 4 The performance of RelNNs and GNNs on mutagenesis benchmark. The columns display: the label content; the architecture that produces the best performance on validation set; the accuracies achieved at the end of the 500 training epochs and those achieved by the network that, during learning, has the best performance on validation set. The architecture is defined by the number of hidden neurons, the state dimension, the unfolding depth and the transition function, which can be a locally recurrent network (lrc), a fully recurrent network (frc) and the sum of the outputs of a feedforward network (sum)

\begin{tabular}{|c|c|c|c|c|c|c|c|}
\hline \multirow[t]{2}{*}{ Model } & \multirow[t]{2}{*}{ Label content } & \multicolumn{4}{|c|}{ Best architecture } & \multicolumn{2}{|l|}{ Accuracy } \\
\hline & & $\begin{array}{l}\text { State } \\
\text { dim. }\end{array}$ & $\begin{array}{l}\text { Hidden } \\
\text { dim. }\end{array}$ & $\begin{array}{l}\text { Unf. } \\
\text { depth }\end{array}$ & $\begin{array}{l}\text { Trans. } \\
\text { type }\end{array}$ & $\begin{array}{l}500 \\
\text { epochs }\end{array}$ & $\begin{array}{l}\text { Best } \\
\text { on val. }\end{array}$ \\
\hline \multicolumn{8}{|c|}{ Whole dataset } \\
\hline GNN & $\mathrm{AB}$ & 10 & 10 & - & - & $81.74[2.42]$ & 79.13 [1.99] \\
\hline GNN & $\mathrm{AB}+\mathrm{C}$ & 5 & 2 & - & - & $88.12[0.50]$ & $85.51[0.66]$ \\
\hline GNN & $\mathrm{AB}+\mathrm{C}+\mathrm{PS}$ & 10 & 2 & - & - & $87.54[1.00]$ & $86.38[0.25]$ \\
\hline RelNN & $\mathrm{AB}$ & 2 & 10 & 2 & sum & $79.57[2.01]$ & $78.26[0.64]$ \\
\hline RelNN & $\mathrm{AB}+\mathrm{C}$ & 5 & 10 & 2 & sum & $77.10[1.47]$ & $79.57[1.70]$ \\
\hline RelNN & $\mathrm{AB}+\mathrm{C}+\mathrm{PS}$ & 5 & 2 & 2 & sum & $80.87[2.51]$ & $83.04[1.13]$ \\
\hline \multicolumn{8}{|c|}{ Regression-friendly part } \\
\hline GNN & $\mathrm{AB}$ & 10 & 10 & - & - & $80.49[0.81]$ & $79.59[0.63]$ \\
\hline GNN & $\mathrm{AB}+\mathrm{C}$ & 2 & 5 & - & - & $94.83[0.83]$ & $93.61[1.07]$ \\
\hline GNN & $\mathrm{AB}+\mathrm{C}+\mathrm{PS}$ & 2 & 2 & - & - & $95.92[0.32]$ & $93.06[0.93]$ \\
\hline RelNN & $\mathrm{AB}$ & 2 & 10 & 2 & sum & $87.77[2.48]$ & $84.75[1.82]$ \\
\hline RelNN & $\mathrm{AB}+\mathrm{C}$ & 2 & 2 & 1 & sum & $87.77[1.22]$ & $88.30[0.45]$ \\
\hline RelNN & $\mathrm{AB}+\mathrm{C}+\mathrm{PS}$ & 10 & 10 & 1 & sum & $88.30[1.27]$ & $91.49[0.53]$ \\
\hline \multicolumn{8}{|c|}{ Regression-unfriendly part } \\
\hline GNN & $\mathrm{AB}$ & 10 & 5 & - & - & $79.67[2.75]$ & $79.83[1.44]$ \\
\hline GNN & $\mathrm{AB}+\mathrm{C}$ & 2 & 10 & - & - & $95.83[1.44]$ & $89.83[1.61]$ \\
\hline GNN & $\mathrm{AB}+\mathrm{C}+\mathrm{PS}$ & 2 & 10 & - & - & $95.83[1.44]$ & $94.33[1.15]$ \\
\hline RelNN & $\mathrm{AB}$ & 2 & 2 & 2 & sum & $78.57[7.72]$ & $79.37[2.71]$ \\
\hline RelNN & $\mathrm{AB}+\mathrm{C}$ & 5 & 10 & 2 & sum & $70.63[4.64]$ & $80.95[2.71]$ \\
\hline RelNN & $\mathrm{AB}+\mathrm{C}+\mathrm{PS}$ & 5 & 10 & 2 & sum & $73.02[4.26]$ & 80.16 [3.98] \\
\hline
\end{tabular}

The results in Table 4 indicate that GNNs and RelNNs can merge the global information with the local information. In particular, the best performances are achieved when pure graph features $\mathrm{AB}$ are merged with node information $\mathrm{C}$ and $\mathrm{C}+\mathrm{PS}$.

Table 5 compares the performance of the models when different number of hidden neurons and different dimensions of the states are used. The table displays the performance on the whole mutagenesis dataset using the features $\mathrm{AB}+\mathrm{C}+\mathrm{PS}$, similar results were obtained using only the friendly and the unfriendly parts of the benchmark and different sets of features. The results show that even if the number of hidden neurons and the state dimension affect the performance, the impact is not very large on the mutagenesis test set. Actually, this fact can be explained by observing that increasing the dimension of the models would allow to implement more "complex functions" on graphs. On the other hand, here such a capability is probably not exploited, because even if the ideal function that classifies correctly the compounds is complex, such a function is not precisely defined by the current training 
Table 5 The effect on the performance of the architecture. RelNNs and GNNs are evaluated on the whole mutagenesis dataset using the features $\mathrm{AB}+\mathrm{C}+\mathrm{PS}$. The columns display: the architecture whose performance is displayed; the accuracies achieved at the end of the 500 training epochs and those achieved by the model that has the best performance on validation set. The architecture is defined by the number of hidden neurons, the state dimension, the unfolding depth and the transition function, which can be a locally recurrent network (lrc), a fully recurrent network (frc) and the sum of the outputs of a feedforward network (sum)

\begin{tabular}{|c|c|c|c|c|c|c|}
\hline \multicolumn{3}{|c|}{ Architecture } & \multicolumn{2}{|c|}{ Train accuracy } & \multicolumn{2}{|c|}{ Test accuracy } \\
\hline $\begin{array}{l}\text { State } \\
\text { dim. }\end{array}$ & $\begin{array}{l}\text { Hidden } \\
\text { num }\end{array}$ & Type & $\begin{array}{l}500 \\
\text { epochs }\end{array}$ & $\begin{array}{l}\text { Best } \\
\text { on val. }\end{array}$ & $\begin{array}{l}500 \\
\text { epochs }\end{array}$ & $\begin{array}{l}\text { Best } \\
\text { on val. }\end{array}$ \\
\hline
\end{tabular}

GNNs on whole dataset using $\mathrm{AB}+\mathrm{C}+\mathrm{PS}$

\begin{tabular}{rrllll}
\hline 2 & 2 & $89.84[0.15]$ & $86.90[0.50]$ & $87.97[1.76]$ & $87.25[1.40]$ \\
2 & 5 & $90.96[0.18]$ & $87.67[0.57]$ & $88.70[0.43]$ & $87.10[0.91]$ \\
2 & 10 & $91.57[0.12]$ & $87.68[0.31]$ & $88.70[0.43]$ & $86.38[1.09]$ \\
5 & 2 & $90.17[0.10]$ & $87.63[0.27]$ & $87.39[0.43]$ & $85.80[1.40]$ \\
5 & 5 & $91.07[0.53]$ & $88.22[0.23]$ & $88.41[0.66]$ & $86.81[0.25]$ \\
5 & 10 & $91.72[0.39]$ & $87.54[0.07]$ & $87.97[0.66]$ & $86.09[1.51]$ \\
10 & 2 & $89.82[0.34]$ & $87.48[0.34]$ & $87.54[1.00]$ & $86.38[0.25]$ \\
10 & 5 & $90.94[0.13]$ & $87.41[0.08]$ & $88.99[0.91]$ & $87.10[1.53]$ \\
10 & 10 & $91.56[0.68]$ & $88.08[0.44]$ & $89.13[0.75]$ & $86.23[1.09]$ \\
\hline
\end{tabular}

RelNNs on whole dataset using $\mathrm{AB}+\mathrm{C}+\mathrm{PS}$

\begin{tabular}{|c|c|c|c|c|c|c|}
\hline 2 & 2 & lrc & 89.62 & 83.37 & 81.01 & 80.72 \\
\hline 2 & 2 & frc & 89.64 & 87.54 & 81.30 & 81.45 \\
\hline 2 & 2 & sum & 86.81 & 82.54 & 78.70 & 81.01 \\
\hline 2 & 5 & lrc & 94.28 & 83.71 & 76.67 & 81.30 \\
\hline 2 & 5 & frc & 95.49 & 84.24 & 79.13 & 82.17 \\
\hline 2 & 5 & sum & 92.36 & 83.24 & 79.13 & 80.72 \\
\hline 2 & 10 & lrc & 96.88 & 82.64 & 76.38 & 80.58 \\
\hline 2 & 10 & frc & 98.24 & 85.92 & 74.78 & 79.86 \\
\hline 2 & 10 & sum & 94.71 & 85.63 & 77.25 & 78.99 \\
\hline 5 & 2 & lrc & 91.18 & 82.14 & 80.87 & 80.29 \\
\hline 5 & 2 & frc & 89.75 & 86.41 & 80.87 & 83.04 \\
\hline 5 & 2 & sum & 85.43 & 83.55 & 78.26 & 77.97 \\
\hline 5 & 5 & lrc & 95.24 & 89.15 & 80.29 & 81.16 \\
\hline 5 & 5 & frc & 97.26 & 83.93 & 77.83 & 81.45 \\
\hline 5 & 5 & sum & 91.68 & 84.22 & 78.70 & 78.70 \\
\hline 5 & 10 & lrc & 98.15 & 88.91 & 76.81 & 82.61 \\
\hline 5 & 10 & $\mathrm{frc}$ & 99.31 & 84.67 & 76.38 & 81.74 \\
\hline 5 & 10 & sum & 95.07 & 84.67 & 78.55 & 80.72 \\
\hline 10 & 2 & lrc & 90.25 & 87.21 & 81.30 & 81.30 \\
\hline 10 & 2 & frc & 90.14 & 86.39 & 78.99 & 81.30 \\
\hline 10 & 2 & sum & 76.18 & 74.35 & 71.88 & 71.88 \\
\hline 10 & 5 & lrc & 95.05 & 85.07 & 79.28 & 81.88 \\
\hline 10 & 5 & frc & 96.92 & 88.03 & 78.99 & 82.46 \\
\hline 10 & 5 & sum & 84.20 & 80.20 & 76.09 & 76.23 \\
\hline 10 & 10 & lrc & 98.39 & 84.91 & 77.68 & 82.46 \\
\hline 10 & 10 & frc & 99.37 & 84.31 & 75.65 & 80.43 \\
\hline 10 & 10 & sum & 91.74 & 85.38 & 77.68 & 79.13 \\
\hline
\end{tabular}


dataset that contains very few patterns. Thus, when the number of the parameters increase, only the performance on training set eventually improves, e.g., in RelNNs.

Moreover, differently from the experiments on the aggregation function problems, the best performances are achieved implementing the transition by recurrent networks (either locally recurrent (lrc) or fully recurrent (frc)) instead of a sum of the outputs of a feedforward network (sum). Such a difference may be due to the fact that the complexity of this problem allows to exploit the larger approximation capability of the recurrent networks.

On the other hand, using the feature set $\mathrm{AB}$ allows us to compare the representations of Figs. 8(a) and (b) in a particular case. In fact, representations (a) and (b) differ both for the graph connectivity and for the label content. But, when $\mathrm{AB}$ is used, the atom labels have the same content both in (a) and (b). The performance of GNNs and RelNNs are closer in this case, which may suggest that the better GNN results may be due also to the different labels used by the two models.

A review of the published results on the "regression-friendly" part can be found in Lodhi and Muggleton (2005), whereas Ramon (2002), Uwents and Blockeel (2005) presents a selection of results using the full set of compounds. Tables $6,7,8$ report the performance achieved by the state of the art techniques on the regression-friendly part, the regressionunfriendly part and the whole mutagenesis dataset, respectively. In order to simplify the comparison, those tables contain also a copy of the best results of RelNNs and GNNs.

The comparison is not straightforward because different methods exploit different feature sets. However, if we focus on the absolute best performance of each method disregarding the used features, we can observe that GNNs outperform other methods on the regressionunfriendly part, while on the friendly part and on the whole dataset the results are close to the state of the art. RelNNs produce slightly lower results with respect to GNNs. On the other hand, if we take in consideration also the features, GNNs and RelNN performance is comparable or better than the other approaches except for the feature set $\mathrm{AB}$. This fact can be explained by noticing that the capability of combining symbolic and sub-symbolic information is an important characteristic of the proposed models. Such a characteristic is less used when the features set does not contain the global properties, so that, in this case, the models lose one of their advantages.

Finally, it is interesting to note that, whereas most of the approaches show a higher level of accuracy on the whole dataset than on the regression-unfriendly part, this is not true for our approaches. Such a behavior may suggest that the proposed connectionist models can capture particular characteristics of the dataset, which cannot be captured by other methods. Those characteristics, however, may not be homogeneously distributed in friendly and unfriendly parts. Such an odd distribution causes a difficulty in learning and gives rise to a decrease in the performance when both parts are used.

\subsection{The biodegradability dataset}

In this section, the experimentation carried out on the biodegradability dataset (Dzeroski 1999; Dzeroski et al. 1999) is presented. Since the experimental procedure is very similar to that adopted for mutagenesis, in the following we only discuss the differences. The reader is referred to the previous section for the representation of the compounds, the model architectures and any other detail that is not explicitly reported here.

The biodegradability dataset is very similar to the mutagenesis dataset. The aim is to predict the degree of biodegradability of 328 chemical compounds. The compounds are described by some global information, molecular weight and $\log \mathrm{P}$, and by the atoms and bonds that constitute them. For atoms and bonds, the type of atom or bond is given. This information gives a full description of the molecules, but in earlier experiments conducted on this 
Table 6 A comparison of the performance of GNNs, RelNNs with other techniques on the regressionfriendly part of the mutagenesis dataset

\begin{tabular}{llll}
\hline Model & Label content & Reference & Accuracy \\
\hline GNN & AB & & 80.49 \\
GNN & AB+C & & 94.83 \\
GNN & AB+C+PS & & 95.92 \\
RelNN & AB & & 84.75 \\
RelNN & AB+C & & 88.30 \\
RelNN & AB+C+PS & & 91.49 \\
RS & AB & Lodhi and Muggleton (2005) & 88.9 \\
RDBC & KB & Ramon (2002) & 84 \\
$1 n n\left(d_{m}\right)$ & $\mathrm{AB}$ & Quinlan and Cameron-Jones (1993) & 83 \\
FOIL & $\mathrm{AB}$ & Kramer and De Raedt (2001) & 76 \\
MFLOG & $\mathrm{AB}+\mathrm{C}$ & Ramon (2002) & 95.7 \\
1 nn $\left(d_{m}\right)$ & $\mathrm{AB}+\mathrm{C}$ & Kirsten (2002) & 91 \\
RDBC & $\mathrm{AB}+\mathrm{C}$ & Srinivasan et al. (1994) & 83 \\
P-Progol & $\mathrm{AB}+\mathrm{C}$ & Lodhi and Muggleton (2005) & 82.0 \\
RS & $\mathrm{AB}+\mathrm{FG}$ & Srinivasan et al. (1994) & 89.9 \\
Neural Networks & $\mathrm{C}+\mathrm{PS}$ & Krogel et al. (2003) & 89.0 \\
RSD & $\mathrm{AB}+\mathrm{C}+\mathrm{FG}$ & Krogel et al. (2003) & 92.6 \\
RELAGGS & $\mathrm{AB}+\mathrm{C}+\mathrm{FG}$ & Srinivasan et al. (1994) & 88.0 \\
P-Progol & $\mathrm{AB}+\mathrm{C}+\mathrm{FG}$ & Krogel et al. (2003) & 88.0 \\
SINUS & $\mathrm{AB}+\mathrm{C}+\mathrm{FG}$ & Lodhi and Muggleton (2005) & 84.5 \\
RS & $\mathrm{AB}+\mathrm{C}+\mathrm{PS}+\mathrm{FG}$ & Quinlan (1996) & 95.8 \\
boosted-FOIL & not available & & 88.3 \\
\hline & & &
\end{tabular}

dataset, extra descriptors were built. These extra descriptors include a vector of occurrences of functional groups and the counts of small substructures in the molecules.

Thus five different features can be used for learning. Three of them are global values and are related to the whole molecule: P0 contains the molecular weight and logP; P1 consists of the counts of the different types of functional groups in the molecule; P2 consists of the counts of common substructures in the molecules. The other two features describe the atoms: $\mathrm{R} 0$ includes atom and bond type; R1 consists of background predicates on the functional groups and substructures. A more detailed description of the information codified by R0, R1, P1 and P2 can be found in Dzeroski et al. (1999).

In our experiments, atom and bond types were represented by one-hot coded vectors, whose length was 11 and 4 . Other features were denoted by their respective values. Thus, P0 was a 2-dimensional vector, P1 and P2 were 30-dimensional sparse vectors.

For this dataset, researchers have studied both the corresponding regression problem, where the half life time has to be predicted and the classification problem, where four categories were defined by common thresholds: chemicals that degrade fast, moderately fast, slowly, or are resistant.

In the classification task, the output networks of RelNNs and GNNs had four output neurons corresponding to the biodegradability classes. A one-hot encoding schema was used to represent each class. The models were trained, by minimization of the common square error function, to return the vector that represents the class to which the processed mole- 
Table 7 A comparison of the performance of GNNs, RelNNs with other techniques on the regressionunfriendly part of the mutagenesis dataset

\begin{tabular}{llll}
\hline Method & Label content & Reference & Accuracy \\
\hline GNN & AB & & 79.67 \\
GNN & AB+C & & 95.83 \\
GNN & AB+C+PS & & 95.83 \\
RelNN & AB & & 79.37 \\
RelNN & AB+C & & 80.95 \\
RelNN & AB+C+PS & & 80.16 \\
TILDE & AB & De Raedt and Blockeel (1997) & 85 \\
RDBC & KB & Kirsten (2002) & 79 \\
$1 n n\left(d_{m}\right)$ & AB & Ramon (2002) & 72 \\
TILDE & AB+C & De Raedt and Blockeel (1997) & 79 \\
RDBC & AB+C & Kirsten (2002) & 79 \\
$1 n n\left(d_{m}\right)$ & $\mathrm{AB}+\mathrm{C}$ & Ramon (2002) & 72 \\
\hline
\end{tabular}

Table 8 A comparison of the performance of GNNs, RelNNs with other techniques on the whole mutagenesis dataset

\begin{tabular}{lllc}
\hline Method & Label content & Reference & Accuracy \\
\hline GNN & AB & & 81.74 \\
GNN & AB+C & & 88.12 \\
GNN & AB+C+PS & & 87.54 \\
RelNN & AB & & 78.26 \\
RelNN & AB+C & & 79.57 \\
RelNN & AB+C+PS & & 83.04 \\
RDBC & KB & Ramon (2002) \\
$1 n n\left(d_{m}\right)$ & AB & De Raedt and Blockeel (1997) & 83 \\
TILDE & AB & Ramon (2002) & 81 \\
$1 n n\left(d_{m}\right)$ & $\mathrm{AB}+\mathrm{C}$ & Kirsten (2002) & 77 \\
RDBC & $\mathrm{AB}+\mathrm{C}$ & De Raedt and Blockeel (1997) & 88 \\
TILDE & $\mathrm{AB}+\mathrm{C}$ & & 82 \\
\hline
\end{tabular}

cule belongs to. In the testing phase, the class predicted by RelNNs and GNNs is the one corresponding to the largest output. The performance was measured by accuracy, i.e., the percentage of the correctly classified compounds, and by accuracy up to one error (accuracy \pm 1 ), which consists of the percentage of examples whose classification is at most one class up or down from the correct classification. For instance, a molecule that belongs to the class fast and is classified as moderately fast, still counts as correctly classified in accuracy \pm 1 .

In the regression task, the RelNN and GNN models, which had only one output, were trained using a real value denoting the degree of biodegradability. Similarly to the original paper (Dzeroski et al. 1999), the performance was evaluated using the correlation score between the output of the model and the target to be predicted. 
Table 9 The performance of RelNNs and GNNs on the biodegradability regression task (at the top) and on the classification task (at the bottom). The performance is measured by the accuracy and the accuracy up to one error (Accuracy \pm 1 ), for the classification task, and by the correlation score, for the regression task

\begin{tabular}{|c|c|c|c|c|c|c|c|c|c|c|c|}
\hline \multicolumn{12}{|c|}{ Regression task } \\
\hline \multirow[t]{2}{*}{ Model } & \multirow{2}{*}{\multicolumn{2}{|c|}{ Label content }} & \multicolumn{5}{|c|}{ Best architecture } & \multicolumn{4}{|c|}{ Correlation } \\
\hline & & & \multirow{2}{*}{$\begin{array}{l}\begin{array}{l}\text { State } \\
\text { dim. }\end{array} \\
10\end{array}$} & \multicolumn{2}{|c|}{$\begin{array}{l}\text { Hidden } \\
\text { num }\end{array}$} & $\begin{array}{l}\text { Unf. } \\
\text { depth }\end{array}$ & $\begin{array}{l}\text { Trans. } \\
\text { type }\end{array}$ & \multicolumn{2}{|l|}{$\begin{array}{l}500 \\
\text { epochs }\end{array}$} & \multicolumn{2}{|c|}{$\begin{array}{l}\text { Best } \\
\text { on val. }\end{array}$} \\
\hline GNN & \multicolumn{2}{|l|}{ R0 } & & 10 & & & & \multicolumn{2}{|c|}{0.6023 [0.0137] } & \multicolumn{2}{|c|}{$0.6035[0.0149]$} \\
\hline GNN & \multicolumn{2}{|l|}{$\mathrm{R} 0+\mathrm{P} 0$} & 5 & 10 & & & & \multicolumn{2}{|c|}{$0.6823[0.0056]$} & \multicolumn{2}{|c|}{$0.6822[0.0049]$} \\
\hline GNN & \multicolumn{2}{|c|}{$\mathrm{R} 0+\mathrm{P} 0+\mathrm{P} 1+\mathrm{P} 2$} & 2 & 10 & & & & \multicolumn{2}{|c|}{$0.4449[0.0411]$} & \multicolumn{2}{|c|}{$0.4452[0.0413]$} \\
\hline RelNN & \multicolumn{2}{|l|}{ R0 } & 5 & 5 & \multicolumn{2}{|c|}{2} & sum & \multicolumn{2}{|c|}{$0.6521[0.0186]$} & \multicolumn{2}{|c|}{$0.6304[0.0140]$} \\
\hline RelNN & \multicolumn{2}{|l|}{$\mathrm{R} 0+\mathrm{P} 0$} & 2 & 5 & \multicolumn{2}{|c|}{2} & sum & \multicolumn{2}{|c|}{$0.6516[0.0203]$} & \multicolumn{2}{|c|}{$0.6499[0.0210]$} \\
\hline RelNN & \multicolumn{2}{|l|}{$\mathrm{R} 0+\mathrm{P} 0+\mathrm{P} 1$} & 5 & 2 & \multicolumn{2}{|c|}{2} & lrc & \multicolumn{2}{|c|}{$0.6066[0.0238]$} & \multicolumn{2}{|c|}{$0.6268[0.0237]$} \\
\hline RelNN & $\mathrm{R} 0+\mathrm{P} 0+\mathrm{P} 2$ & & 5 & 5 & 1 & & $\operatorname{lrc}$ & $0.5619[0$ & $.0427]$ & 0.6 & $5417[0.0120]$ \\
\hline RelNN & $\mathrm{R} 0+\mathrm{P} 0+\mathrm{P} 1+\mathrm{P}$ & & 2 & 5 & 1 & & frc & $0.5287[0$ & $.0244]$ & 0.6 & $5729[0.0213]$ \\
\hline Classifi & ication task & & & & & & & & & & \\
\hline Model & Label content & Best a & architectu & & & Acc & aracy & & Accura & $y \pm 1$ & \\
\hline & & $\begin{array}{l}\text { State } \\
\text { dim. }\end{array}$ & $\begin{array}{l}\text { Hidden } \\
\text { num }\end{array}$ & $\begin{array}{l}\text { Unf. } \\
\text { depth }\end{array}$ & $\begin{array}{l}\text { Trans. } \\
\text { type }\end{array}$ & $\begin{array}{l}500 \\
\text { epoc }\end{array}$ & & $\begin{array}{l}\text { Best } \\
\text { on val. }\end{array}$ & $\begin{array}{l}500 \\
\text { epochs }\end{array}$ & & $\begin{array}{l}\text { Best } \\
\text { on val. }\end{array}$ \\
\hline GNN & R0 & 2 & 10 & & & 52.6 & 0 [0.97] & $45.01[1.36]$ & $89.42[0$ & $.94]$ & $89.01[1.70]$ \\
\hline GNN & $\mathrm{R} 0+\mathrm{P} 0$ & 5 & 10 & & & 58.3 & $4[0.97]$ & $53.66[3.51]$ & $92.96[0$ & $91]$ & $91.65[0.18]$ \\
\hline GNN & $\mathrm{R} 0+\mathrm{P} 0+\mathrm{P} 1$ & 10 & 10 & & & 54.8 & {$[1.31]$} & $42.66[0.63]$ & 91.55 & $.45]$ & 89.60 [1.33] \\
\hline GNN & $\mathrm{R} 0+\mathrm{P} 0+\mathrm{P} 2$ & 2 & 10 & & & 53.3 & 1 [1.63] & 41.68 [1.97] & 88.60[ & .37] & 91.34 [1.53] \\
\hline GNN & $\mathrm{R} 0+\mathrm{P} 0+\mathrm{P} 1+\mathrm{P} 2$ & 2 & 10 & & & 51.0 & 9 [1.85] & 41.17 [0.02] & 87.38[ & $.00]$ & $88.32[1.55]$ \\
\hline RelNN & R0 & 5 & 2 & 2 & $\operatorname{lrc}$ & 39.3 & $3[3.16]$ & 40.00 [1.37] & 87.87[ & .30] & $92.26[0.63]$ \\
\hline RelNN & $\mathrm{R} 0+\mathrm{P} 0$ & 2 & 2 & 1 & frc & 43.2 & $3[1.60]$ & $43.60[0.43]$ & 91.95[ & $.56]$ & $92.99[0.83]$ \\
\hline RelNN & $\mathrm{R} 0+\mathrm{P} 0+\mathrm{P} 1$ & 2 & 5 & 1 & lrc & 52.0 & $1[4.01]$ & $50.73[1.62]$ & 91.04[ & .19] & 92.01 [1.02] \\
\hline RelNN & $\mathrm{R} 0+\mathrm{P} 0+\mathrm{P} 2$ & 5 & 5 & 2 & frc & 48.7 & 2 [2.43] & $47.62[1.47]$ & 88.60[ & $.02]$ & $90.67[0.88]$ \\
\hline RelNN & $\mathrm{R} 0+\mathrm{P} 0+\mathrm{P} 1+\mathrm{P} 2$ & 2 & 5 & 2 & $\operatorname{lrc}$ & 49.9 & $4[1.77]$ & 51.46 [2.09] & 89.39 & .19] & $91.22[0.85]$ \\
\hline
\end{tabular}

Table 9 displays the performance on the biodegradability dataset. The best overall result, both on the classification and the regression tasks, was obtained by GNNs with input R0+P0. In most of the input configurations, GNNs outperform RelNNs, except on regression task when all the features $\mathrm{R} 0+\mathrm{P} 0+\mathrm{P} 1+\mathrm{P} 2$ are used. Interestingly, the performance of both models is maximal when an intermediate configuration is used between the shortest input R0 and the longest $\mathrm{R} 0+\mathrm{P} 0+\mathrm{P} 1+\mathrm{P} 2$. Such a fact can be explained by observing that a larger set of features requires a larger number of parameters and makes the networks more prone to fail in generalization and to have overfitting problems. It is also interesting to notice that the performance of the models is high even without any preprocessed input feature, i.e., using R0, which includes only the graph connectivity and the bond and atom types.

Moreover, differently from the experiments on the mutagenesis, the stopping criterion based on a large number of epochs and the criterion based on validation set achieve close performances. The only exception occurs for GNN accuracy on the classification task: in this case the former criterion clearly outperforms the latter. Notice that, even on the same 
Table 10 The effect on the performance of the number of hiddens and the state dimension. RelNNs and GNNs are evaluated on the classification task of biodegradability using R0+P0. The columns display: the number of hiddens and state dimension of the model whose performance is displayed; the accuracies achieved at the end of the 500 training epochs and those achieved by the model that has the best performance on validation set

\begin{tabular}{|c|c|c|c|c|c|c|c|}
\hline \multicolumn{2}{|c|}{ Architecture } & \multicolumn{2}{|c|}{ Train accuracy } & \multicolumn{2}{|c|}{ Test accuracy } & \multicolumn{2}{|c|}{ Test accuracy \pm 1} \\
\hline State & Hidden & 500 & Best & 500 & Best & 500 & Best \\
\hline dim. & num & epochs & on val. & epochs & on val. & epochs & on val. \\
\hline
\end{tabular}

GNNs on the classification task using R0+P0

\begin{tabular}{rrllllll}
\hline 2 & 2 & $66.96[0.24]$ & $56.33[1.52]$ & $53.11[1.79]$ & $49.10[2.18]$ & $92.26[1.25]$ & $90.22[1.21]$ \\
2 & 5 & $74.57[0.68]$ & $58.03[2.60]$ & $56.79[0.46]$ & $48.27[1.08]$ & $92.16[0.36]$ & $92.25[0.17]$ \\
2 & 10 & $78.39[1.00]$ & $58.86[4.67]$ & $57.09[0.16]$ & $47.48[3.55]$ & $91.64[0.35]$ & $91.03[0.98]$ \\
5 & 2 & $67.03[0.74]$ & $57.75[0.53]$ & $53.33[2.51]$ & $51.42[0.35]$ & $90.22[0.31]$ & $89.61[1.05]$ \\
5 & 5 & $75.61[0.33]$ & $61.06[2.42]$ & $57.82[1.37]$ & $49.97[1.53]$ & $92.56[1.37]$ & $90.92[1.57]$ \\
5 & 10 & $79.52[0.35]$ & $64.58[3.75]$ & $58.34[0.97]$ & $53.66[3.51]$ & $92.96[0.91]$ & $91.65[0.18]$ \\
10 & 2 & $66.75[1.10]$ & $54.72[2.15]$ & $52.93[0.49]$ & $48.87[0.75]$ & $90.63[0.46]$ & $91.02[0.45]$ \\
10 & 5 & $75.71[0.54]$ & $59.96[0.74]$ & $57.30[1.70]$ & $49.49[3.50]$ & $92.46[0.76]$ & $89.80[1.84]$ \\
10 & 10 & $79.12[0.29]$ & $64.71[3.93]$ & $56.71[0.80]$ & $52.64[4.28]$ & $93.17[0.87]$ & $91.35[0.45]$ \\
\hline RelNNs on the classification task using R0+P0 & & & & 95 \\
\hline 2 & 2 & 50.79 & 45.43 & 43.23 & 43.60 & 91.95 & 92.99 \\
2 & 5 & 61.71 & 46.10 & 41.16 & 42.50 & 88.29 & 93.66 \\
2 & 10 & 69.43 & 47.33 & 42.87 & 43.29 & 86.28 & 91.95 \\
5 & 2 & 51.49 & 46.33 & 43.60 & 43.54 & 91.71 & 93.66 \\
5 & 5 & 62.82 & 46.06 & 42.68 & 43.96 & 87.80 & 93.23 \\
5 & 10 & 73.40 & 45.56 & 39.02 & 42.50 & 85.85 & 93.05 \\
10 & 2 & 52.23 & 46.18 & 42.56 & 42.93 & 92.62 & 93.60 \\
10 & 5 & 65.71 & 47.93 & 44.39 & 43.17 & 88.66 & 92.99 \\
10 & 10 & 78.82 & 46.62 & 39.09 & 41.71 & 85.67 & 92.68 \\
\hline
\end{tabular}

task, observing the accuracy \pm 1 , we cannot identify a method that is clearly preferable. This event may be explained observing that the square error function used during training tends to directly increase the accuracy, whereas the impact on accuracy \pm 1 is an expected consequence. Such a difference may indirectly changes the performance of the two stopping criteria.

Table 10 illustrates the effect of using different numbers of hidden neurons and state dimensions. ${ }^{28}$ It can be noticed that distance between the accuracy on the training set and the accuracy on the test set increases in GNNs when a larger number of parameters are employed. Such a behaviour confirms the presence of an overfitting problem that does not allow to improve the performance with larger inputs. It is worth mentioning that the same

\footnotetext{
${ }^{28}$ For sake of brevity, the table shows only the performance on the classification task using the features $\mathrm{R} 0+\mathrm{P} 0$, which is a case in the middle between the minimal number of features in $\mathrm{R} 0$ and the maximal in $\mathrm{R} 0+\mathrm{P} 0+\mathrm{P} 1+\mathrm{P} 2$. Moreover, for RelNNs, only the "sum" transition function is considered. However, the other cases showed similar results with respect to the goal of analyzing the effect of the number of hiddens and of state dimension.
} 
Table 11 A comparison of the performance of GNNs and RelNN with other methods on the classification task of the biodegradability benchmark

\begin{tabular}{|c|c|c|c|}
\hline Models & Label content & Accuracy & Accuracy \pm 1 \\
\hline GNN & R0 & 52.60 & 89.42 \\
\hline GNN & $\mathrm{R} 0+\mathrm{P} 0$ & 58.34 & 92.96 \\
\hline GNN & $\mathrm{R} 0+\mathrm{P} 0+\mathrm{P} 1$ & 54.85 & 91.55 \\
\hline GNN & $\mathrm{R} 0+\mathrm{P} 0+\mathrm{P} 2$ & 53.31 & 88.60 \\
\hline GNN & $\mathrm{R} 0+\mathrm{P} 0+\mathrm{P} 1+\mathrm{P} 2$ & 51.09 & 87.38 \\
\hline RelNN & R0 & 35.57 & 85.47 \\
\hline RelNN & $\mathrm{R} 0+\mathrm{P} 0$ & 36.59 & 88.11 \\
\hline RelNN & $\mathrm{R} 0+\mathrm{P} 0+\mathrm{P} 1$ & 42.68 & 91.16 \\
\hline RelNN & $\mathrm{R} 0+\mathrm{P} 0+\mathrm{P} 2$ & 40.65 & 90.96 \\
\hline RelNN & $\mathrm{R} 0+\mathrm{P} 0+\mathrm{P} 1+\mathrm{P} 2$ & 44.11 & 89.02 \\
\hline $\mathrm{C} 4.5$ & $\mathrm{P} 0+\mathrm{P} 1$ & 55.2 & 86.2 \\
\hline $\mathrm{C} 4.5$ & $\mathrm{P} 0+\mathrm{P} 2$ & 56.9 & 82.4 \\
\hline RIPPER & $\mathrm{P} 0+\mathrm{P} 1$ & 52.6 & 89.8 \\
\hline RIPPER & $\mathrm{P} 0+\mathrm{P} 2$ & 57.6 & 93.9 \\
\hline M5' & $\mathrm{P} 0+\mathrm{P} 1$ & 53.8 & 94.5 \\
\hline M5' & $\mathrm{P} 0+\mathrm{P} 2$ & 59.8 & 94.7 \\
\hline FFOIL & $\mathrm{P} 0+\mathrm{R} 0$ & 53.0 & 88.7 \\
\hline ICL & $\mathrm{P} 0+\mathrm{R} 1$ & 55.7 & 92.6 \\
\hline SRT-C & $\mathrm{P} 0+\mathrm{P} 1$ & 50.8 & 87.5 \\
\hline SRT-C & $\mathrm{P} 0+\mathrm{P} 1+\mathrm{R} 1$ & 55.0 & 90.0 \\
\hline SRT-R & $\mathrm{P} 0+\mathrm{P} 1$ & 49.5 & 91.9 \\
\hline SRT-R & $\mathrm{P} 0+\mathrm{P} 1+\mathrm{R} 1$ & 51.6 & 92.8 \\
\hline TILDE-C & $\mathrm{P} 0+\mathrm{R} 1$ & 51.0 & 88.6 \\
\hline TILDE-C & $\mathrm{P} 0+\mathrm{P} 1+\mathrm{R} 1$ & 52.0 & 89.0 \\
\hline TILDE-R & $\mathrm{P} 0+\mathrm{R} 1$ & 52.6 & 94.0 \\
\hline TILDE-R & $\mathrm{P} 0+\mathrm{P} 1+\mathrm{R} 1$ & 52.4 & 93.9 \\
\hline
\end{tabular}

phenomenon, which is not evident on the R0+P0, arises also in RelNNs when larger inputs are used.

Tables 11, 12 show the results achieved on the classification task and on the regression task, respectively, as published in the literature (Dzeroski et al. 1999). Notice that R0, which contains the graph connectivity, is needed by GNNs and RelNNs and has been always used in our experiments, whereas such information is not useful or even misleading for most of the other methods, since R0 is partially and implicitly contained in the other features. The tables prove that the performance of our models is comparable to the other best results, but not better: GNNs achieve the second best performance on classification task accuracy and on regression task. The high results obtained by methods that do not exploit R0 suggests that the counts of functional groups and substructures are probably a good propositionalization of the relational data (graph connectivity), as already mentioned in Dzeroski et al. (1999). On the other hand, the relatively high performance obtained by the presented approaches using only R0 suggest that those methods can actually extract a large part of the information in P0, P1, P2, R1 directly from the original graph. 
Table 12 A comparison of the performance of GNNs and RelNN with other methods on the regression task of the biodegradability benchmark

\begin{tabular}{lll}
\hline Models & Label content & Correlation \\
\hline GNN & R0 & 0.6023 \\
GNN & R0+P0 & 0.6823 \\
GNN & R0+P0+P1+P2 & 0.4449 \\
RelNN & R0 & 0.6511 \\
RelNN & R0+P0 & 0.6329 \\
RelNN & R0+P0+P1 & 0.6253 \\
RelNN & R0+P0+P2 & 0.5228 \\
RelNN & R0+P0+P1+P2 & 0.5515 \\
M5 ${ }^{\prime}$ & P0+P1 & 0.666 \\
M5' & P0+P2 & 0.693 \\
SRT-R & P0+P1 & 0.580 \\
SRT-R & P0+P1+R1 & 0.632 \\
TILDE-R & P0+R1 & 0.622 \\
TILDE-R & P0+P1+R1 & 0.623 \\
\hline
\end{tabular}

\section{Conclusions}

In this paper, we described and studied two recently proposed connectionist methods for graph processing called Graph Neural Networks and Relational Neural Networks. The methods were compared and experimentally evaluated on benchmarks commonly used in relational learning field of research. The results are promising and suggest that RelNNs and GNNs can be viable approaches for learning on relational data. In particular, on mutagenesis, the performance of RelNNs and GNNs equals and, in some cases, outperforms the state of the art.

A larger experimentation of the proposed models is matter of future research. In particular, the fields of bioinformatics and image localization appear to provide applications where GNNs and RelNNs may be useful. Moreover, a number of extensions of the models can be investigated. For example, it may be interesting to study how the models can cope with dynamic information, i.e., input graphs that change along time, or how they can deal with missing information, for instance, label fields that are not available, and how the missing information can be eventually predicted. Moreover, several studies, which have been already carried out for common feedforward networks, should be extended to GNNs and RelNNs. For instance, the concept of Vapnik-Chervonenkis dimension and the cases when learning without local minima is possible have not been investigated for the proposed neural models.

\section{References}

Almeida, L. B. (1990). A learning rule for asynchronous perceptrons with feedback in a combinatorial environment. Piscataway: IEEE Press. pp. 102-111.

Back, A., \& Tsoi, A. C. (1994). Locally recurrent globally feedforward networks, a critical review of architectures. IEEE Transactions on Neural Networks, 5(3), 229-239.

Baldi, P., \& Pollastri, G. (2004). The principled design of large-scale recursive neural network architecturesDAG-RNNs and the protein structure prediction problem. Journal of Machine Learning Research, 4, 575-602. 
Bengio, Y., Frasconi, P., \& Simard, P. (1994). Learning long-term dependencies with gradient descent is difficult. IEEE Transactions on Neural Networks, 5, 157-166. Special Issue on Recurrent Neural Networks.

Bianchini, M., Gori, M., \& Scarselli, F. (2001). Processing directed acyclic graphs with recursive neural networks. IEEE Transactions on Neural Networks, 12(6), 1464-1470.

Bianchini, M., Mazzoni, P., Sarti, L., \& Scarselli, F. (2003). Face localization with recursive neural networks. In B. Apolloni, M. Marinaro, \& R. Tagliaferri (Eds.), Lecture notes in computer science: Vol. 2859. Proceedings of WIRN03 (pp. 99-105). Berlin: Springer.

Bianchini, M., Maggini, M., Sarti, L., \& Scarselli, F. (2005). Recursive neural networks for processing graphs with labelled edges: Theory and applications. Neural Networks-Special Issue on Neural Networks and Kernel Methods for Structured Domains, 18(8), 1040-1050.

Bianchini, M., Gori, M., Sarti, L., \& Scarselli, F. (2006). Recursive processing of cyclic graphs. IEEE Transactions on Neural Networks, 17(1), 10-18.

Blockeel, H., \& Bruynooghe, M. (2003). Aggregation versus selection bias, and relational neural networks. In IJCAI-2003 workshop on learning statistical models from relational data, SRL-2003 (Vol. 11).

Bloehdorn, S., \& Blohm, S. (2006). A self organizing map for relation extraction from wikipedia using structured data representations. In Proceedings of the international workshop on intelligent information access, IIIA-2006. Berlin: Springer.

Brin, S., \& Page, L. (1998). The anatomy of a large-scale hypertextual Web search engine. Computer Networks and ISDN Systems, 30(1-7), 107-117.

Chang, H., Cohn, D., \& McCallum, A. (2000). Learning to create customized authority lists. In Proceedings of the 17th international conference on machine learning, ICML 'OO (pp. 127-134). San Mateo: Morgan Kaufmann.

Chapelle, O., Schölkopf, B., \& Zien, A. (Eds.) (2006). Semi-supervised learning. Cambridge: MIT Press.

De Raedt, L., \& Blockeel, H. (1997). Using logical decision trees for clustering. In Lecture notes in artificial intelligence: Vol. 1297. Proceedings of the 7th international workshop on inductive logic programming ILP-97 (pp. 133-141). Berlin: Springer.

Debnath, A., Lopex de Compandre, R., Debnath, G., Schusterman, A., \& Hansch, C. (1991). Structureactivity relationship of mutagenic aromatic and heteroaromatic nitro compounds. Correlation with molecular orbital energies and hydrophobicity. Journal of Medicinal Chemistry, 34(2), 786-797.

Di Massa, V., Monfardini, G., Sarti, L., Scarselli, F., Maggini, M., \& Gori, M. (2006). A comparison between recursive neural networks and graph neural networks. In International joint conference on neural networks (pp. 778-785).

Dzeroski, S. (1999). Biodegradability dataset, info at: http://www-ai.ijs.si/ilpnet2/apps/pbiodeg.html.

Dzeroski, S., Blockeel, H., Kompare, B., Kramer, S., Pfahringer, B., \& Van Laer, W. (1999). Experiments in predicting biodegradability. In Lecture notes in computer science: Vol. 1634. Proceedings of the 9th international workshop on inductive logic programming, ILP-99 (pp. 80-91).

Francesconi, E., Frasconi, P., Gori, M., Marinai, S., Sheng, J., Soda, G., \& Sperduti, A. (1998). Logo recognition by recursive neural networks. In K. Tombre \& A. K. Chhabra (Eds.), GREC '97: Selected papers from the second international workshop on graphics recognition, algorithms and systems (pp. 104-117). Berlin: Springer.

Frasconi, P., Gori, M., \& Sperduti, A. (1998). A general framework for adaptive processing of data structures. IEEE Transactions on Neural Networks, 9(5), 768-786.

Gärtner, T. (2003). Kernel-based learning in multi-relational data mining. ACM SIGKDD Explorations, 5(1), 49-58.

Gärtner, T., Lloyd, J., \& Flach, P. (2004). Kernels and distances for structured data. Machine Learning, 57(3), 205-232.

Getoor, L., \& Taskar, B. (2007). Introduction to statistical relational learning. Cambridge: MIT Press.

Goller, C. (1997). A connectionist approach for learning search control heuristics for automated deduction systems. PhD thesis, Technische Universität München.

Goller, C., \& Küchler, A. (1996). Learning task-dependent distributed representations by backpropagation through structure. IEEE Transactions on Neural Networks, 1, 347-352.

Gori, M., Scarselli, F., \& Tsoi, A. C. (1998). On the closure of set of functions that can be realized by a multilayer perceptron. IEEE Transactions on Neural Networks, 9(6), 1086-1098.

Gori, M., Monfardini, G., \& Scarselli, F. (2005). A new model for learning in graph domains. In Proceedings international joint conference on neural networks (IJCNN2005) (Vol. 2, pp. 729-734).

Goulon-Sigwalt-Abram, A., Duprat, A., \& Dreyfus, G. (2005). From hopfield nets to recursive networks to graph machines: numerical machine learning for structured data. Theoretical Computer Science, 344(23), 298-334.

Hagenbuchner, M., Sperduti, A., \& Tsoi, A. C. (2003). A self-organizing map for adaptive processing of structured data. IEEE Transactions on Neural Networks, 14(3), 491-505. 
Hagenbuchner, M., Sperduti, A., \& Tsoi, A. (2005). Contextual self-organizing maps for structured domains. In Lecture notes in computer science: Vol. 3720. Proceedings of the workshop on relational machine learning, 16th European conference on machine learning ECML 2005 (pp. 46-55). Berlin: Springer.

Hagenbuchner, M., Sperduti, A., Tsoi, A. C., Trentini, F., Scarselli, F., \& Gori, M. (2006). Clustering XML documents using self-organizing maps for structures. In Lecture notes in computer science: Vol. 3977. Advances in XML information retrieval and evaluation (pp. 481-496). Berlin: Springer.

Hagenbuchner, M., Sperduti, A., \& Tsoi, A. C. (2009). Graph self-organizing maps for cyclic and unbounded graphs. Neurocomputation, 72(7-9), 1419-1430.

Hammer, B. (1999). Approximation capabilities of folding networks. In M. Caudill \& C. Butler (Eds.), Proceedings of the 7th European symposium on artificial neural networks, ESANN 1999 (pp. 33-38).

Hammer, B., \& Jain, J. (2004). Neural methods for non-standard data. In M. Verleysen (Ed.), Proceedings of the 12th European symposium on artificial neural networks, ESANN 2004 (pp. 281-292). D-side publications.

Hammer, B., Micheli, A., Sperduti, A., \& Strickert, M. (2004a). A general framework for unsupervised processing of structured data. Neurocomputing, 57, 3-35.

Hammer, B., Micheli, A., Sperduti, A., \& Strickert, M. (2004b). Recursive self-organizing network models. Neural Networks, 17(8-9), 1061-1085.

Haykin, S. (1994). Neural networks: A comprehensive foundation. New York: Prentice Hall.

Jensen, F. V. (1996). Introduction to Bayesian networks. Berlin: Springer.

Khamsi, M. A. (2001). An introduction to metric spaces and fixed point theory. New York: Wiley.

Kirsten, M. (2002). Multirelational distance-based clustering. PhD thesis, School of Computer Science, Ottovon-Guericke University, Magdeburg, Germany.

Kleinberg, J. (1999). Authoritative sources in a hyperlinked environment. Journal of the ACM, 46(5), 604632.

Kondor, R., \& Lafferty, J. (2002). Diffusion kernels on graphs and other discrete structures. In C. Sammut \& A. G. Hoffmann (Eds.), Proceedings of the 19th international conference on machine learning, ICML 2002 (pp. 315-322). San Mateo: Morgan Kaufmann.

Krahmer, E., Erk, S., \& Verleg, A. (2003). Graph-based generation of referring expressions. Computational Linguistics, 29(1), 53-72.

Kramer, S., \& De Raedt, L. (2001). Feature construction with version spaces for biochemical applications. In Proceedings of the 18th international conference on machine learning, ICML 2001 (pp. 258-265). San Mateo: Morgan Kaufmann.

Krogel, M., Rawles, S., Zelezny, F., Flach, P., Lavrac, N., \& Wrobel, S. (2003). Comparative evaluation of approaches to propositionalization. In Lecture notes in computer science: Vol. 2835. Proceedings of the 13th international conference on inductive logic programming, ILP 2003 (pp. 197-214). Berlin: Springer.

Lafferty, J., McCallum, A., \& Pereira, F. (2001). Conditional random fields: Probabilistic models for segmenting and labeling sequence data. In Proceedings of 18th international conference on machine learning.

Lodhi, H., \& Muggleton, S. H. (2005). Is Mutagenesis still challenging? In Proceedings of the 15th international conference on inductive logic programming, ILP 2005, late-breaking papers (pp. 35-40). Cambridge: MIT Press.

McClelland, J., Rumelhart, D., \& the PDP Research Group (1986). Parallel distributed processing: explorations in the microstructure of cognition (Vol. 2). Cambridge: MIT Press.

Micheli, A. (2009). Neural network for graphs: A contextual constructive approach. IEEE Transactions on Neural Networks, 20(3), 498-511.

Micheli, A., Sperduti, A., Starita, A., \& Bianucci, A. M. (2001). Analysis of the internal representations developed by neural networks for structures applied to quantitative structure-activity relationship studies of benzodiazepines. Journal of Chemical Information and Computer Sciences, 41(2), 202-218.

Micheli, A., Sona, D., \& Sperduti, A. (2004). Contextual processing of structured data by recursive cascade correlation. IEEE Transactions on Neural Networks, 15(6), 1396-1410.

Money, C., \& Pollastri, G. (2009). Beyond the twilight zone: Automated prediction of structural properties of protein by recursive neural networks and remote homology information. Proteins: Structure, Function, and Bioinformatics, 77(1), 181-190.

Monfardini, G., \& Scarselli, F. (2004). The graph neural networks toolbox, freely available for download at http://airgroup.dii.unisi.it/projects/GraphNeuralNetwork/download.htm.

Mutagenesis (1991). Mutagenesis dataset, available for download at http:/www.comlab.ox.ac.uk/activities/ machinelearning/mutagenesis.html.

Newman, M. E. J. (2001). From the cover: The structure of scientific collaboration networks. Proceedings National Academy of Sciences, 98(2), 404-409.

Pineda, F. (1987). Generalization of back-propagation to recurrent neural networks. Physical Review Letters, 59, 2229-2232. 
Quinlan, J. R. (1996). Boosting first-order learning. In S. Arikawa \& A. Sharma (Eds.), Lecture notes in computer science: Vol. 1160. Proceedings of the 7th international workshop on algorithmic learning theory, ALT 1996 (p. 143). Berlin: Springer.

Quinlan, J., \& Cameron-Jones, R. (1993). FOIL: A midterm report. In Proceedings of the European conference on machine learning (pp. 3-20).

Ramon, J. (2002). Clustering and instance based learning in first order logic. PhD thesis, K.U. Leuven, Belgium.

Riedmiller, M., \& Braun, H. (1993). A direct adaptive method for faster backpropagation learning: the RPROP algorithm. In Proceedings of the IEEE international conference on neural networks (Vol. 1, pp. 586-591). San Francisco, CA, USA.

Rodriguez, P. (2001). Simple recurrent networks learn context-free and context-sensitive languages by counting. Neural Computation, 13, 2093-2118.

Scarselli, F., Yong, S., Gori, M., Hagenbuchner, M., Tsoi, A., \& Maggini, M. (2005). Graph neural networks for ranking web pages. In Proceedings of the 2005 IEEE/WIC/ACM conference on web intelligence, WI2005 (pp. 666-672), Washington, DC, USA. Washington: IEEE Computer Society.

Scarselli, F., Gori, M., Monfardini, G., Tsoi, A. C., \& Hagenbuchner, M. (2009a). Computational capabilities of graph neural networks. IEEE Transactions on Neural Networks, 20(1), 81-102.

Scarselli, F., Gori, M., Monfardini, G., Tsoi, A. C., \& Hagenbuchner, M. (2009b). The graph neural network model. IEEE Transactions on Neural Networks, 20(1), 61-80.

Sperduti, A. (1994). Labelling RAAM. Connection Science, 6(4), 429-459.

Sperduti, A., \& Starita, A. (1997). Supervised neural networks for the classification of structures. IEEE Transactions on Neural Networks, 8(3), 714-735.

Srinivasan, A., Muggleton, S., King, R., \& Sternberg, M. (1994). Mutagenesis: ILP experiments in a nondeterminate biological domain. In S. Wrobel (Ed.), GMD-Studien: Vol. 23. Proceedings of the 4th international workshop on inductive logic programming, ILP 1994 (pp. 217-232). Gesellschaft für Mathematik und Datenverarbeitung $\mathrm{MBH}$.

Sturt, P., Costa, F., Lombardo, V., \& Frasconi, V. (2003). Learning first-pass structural attachment preferences with dynamic grammars and recursive neural networks. Cognition, 88(2), 133-169.

Tsoi, A. C., Morini, G., Scarselli, F., Hagenbuchner, M., \& Maggini, M. (2003). Adaptive ranking of web pages. In Proceedings of the 12th international conference on world wide web, WWW 2003, New York, NY, USA. New York: ACM.

Tsoi, A. C., Hagenbuchner, M., \& Scarselli, F. (2006). Computing customized page ranks. ACM Transactions on Internet Technology, 6(4), 381-414.

Uwents, W., \& Blockeel, H. (2005). Classifying relational data with neural networks. In Lecture notes in computer science: Vol. 3625. Proceedings of the 15th international conference on inductive logic programming, ILP 2005 (pp. 384-396). Berlin: Springer.

Vapnik, V. N. (1998). Statistical learning theory. New York: Wiley.

Wang, Z., Hagenbuchner, M., Tsoi, A., Cho, S. Y., \& Chi, Z. (2002). Image classification with structured self-organization map. In Proceedings of the 2002 international joint conference on neural networks, IJCNN 2002 (Vol. 2, pp. 1918-1923), Piscataway, NJ, USA. New York: IEEE Press.

Werbos, P. (1990). Backpropagation through time: what it does and how to do it. Proceedings of the IEEE, 78(10), 1550-1560.

Yong, S., Hagenbuchner, M., Scarselli, F., Tsoi, A. C., \& Gori, M. (2006). Document mining using graph neural networks. In N. Fuhr, M. Lalmas, \& A. Trotman (Eds.), Lecture notes in computer science: Vol. 4518. Proceedings of the 5th international workshop of the initiative for the evaluation of XML retrieval, INEX 2006, revised and selected papers (pp. 458-472). Berlin: Springer. 